\title{
ENVIRONMENTALLY RESPONSIBLE FOOD CHOICE: CHARACTERISTICS AND SUMMARIES OF STUDIES
}

The following Tables present selected characteristics of relevant studies on environmentallyresponsible food choice, classified in five broad categories. Table B.1.1 provides a summary of "general reviews" of the literature. Table B.1.2 presents studies on the perception of environmentally responsible products. Table B.1.3 reviews empirical studies using real market data/experimental economics. Table B.1.4 includes studies focussing on hypothetical markets (e.g. willingness to pay). Table B.1.5 presents studies available on the perception of labels.

The next Tables summarise the main results from selected papers on environmentally responsible food choice providing significant contributions in terms of methodology or results. Only the effects of relevant and significant explanatory variables are reported. Table B.2.1 provides results from studies using real market data/experimental economics. Table B.2.2 provides results from studies focussing on hypothetical markets (e.g. willingness to pay). In these Tables, the reference product is indicated in bold character and the description of dependent variable is indicated in italics. 
TABLE B.1.1: Overview of general reviews of the "environmentally-responsive food choice" literature

\begin{tabular}{|c|c|c|c|}
\hline Authors & Year & Purpose of the study & Explanatory variables \\
\hline Brennan C., Gallagher K. and McEachern M & 2003 & $\begin{array}{l}\text { Provides a critical review of the literature on the consumer interest in the UK in organic food, } \\
\text { with a particular focus on organic meat }\end{array}$ & \\
\hline Harris B., Burress D. and Eicher S. & 2000 & $\begin{array}{l}\text { Reviews articles investigating the characteristics and preferences of consumers of, and } \\
\text { demand for, Environmentally Identified Products in the US }\end{array}$ & $\begin{array}{l}\text { Summary of main variables } \\
\text { affecting consumers' } \\
\text { preferences for EIPs }\end{array}$ \\
\hline $\begin{array}{l}\text { Midmore P., Naspetti S., Sherwood A.-M., Vairo D., } \\
\text { Wier M. and Zanoli R., }\end{array}$ & 2005 & $\begin{array}{l}\text { Produced on the basis of a workshop (held in September 2004) convened to discuss the } \\
\text { relevance of consumer research to the wider "QualityLowInputFood (QLIF) European } \\
\text { integrated project". It has three aims: to report on a survey and analysis of both scientific } \\
\text { literature and also recent primary data available to the project team providing insights to } \\
\text { consumer attitudes to organic products; to support new empirical investigations of consumer } \\
\text { attributes which will be the focus in later stages of Sub-Project 1; and, based on the foregoing, } \\
\text { to raise issues for broader discussion }\end{array}$ & \\
\hline Parker Wheatley W. & 2001 & $\begin{array}{l}\text { Reviews the literature on consumer preferences and willingness to pay for pork produced } \\
\text { through alternative methods such as natural and organic production. Investigates the degree to } \\
\text { which such premiums are transmitted to pork producers }\end{array}$ & $\begin{array}{lr}\begin{array}{l}\text { Cost of } \\
\text { production }\end{array} & \text { alternative } \\
\text { compared to WTP } & \text { methods }\end{array}$ \\
\hline Thompson G.D. & 1998 & $\begin{array}{l}\text { Reviews consumer demand studies specifying organic products analysed, geographic scope, } \\
\text { purpose of the study, survey method, sample size }\end{array}$ & $\begin{array}{l}\text { Price elasticities, income, } \\
\text { store effects, age, gender, } \\
\text { marital status, education, } \\
\text { household size }\end{array}$ \\
\hline
\end{tabular}


TABLE B.1.2: Perception of environmentally-responsible products

\begin{tabular}{|c|c|c|c|c|c|c|}
\hline Authors & Year & Purpose of the study & $\begin{array}{l}\text { Survey } \\
\text { method }\end{array}$ & Methodology & $\begin{array}{l}\text { Dependent } \\
\text { variable(s) }\end{array}$ & Explanatory variables \\
\hline $\begin{array}{l}\text { Anderson M.D. et } \\
\text { al. }\end{array}$ & 1996 & $\begin{array}{l}\text { Examines consumer awareness of } \\
\text { integrated pest management (IPM) and the } \\
\text { effects of two marketing strategies (active } \\
\text { and passive) }\end{array}$ & $\begin{array}{l}\text { Direct interviews } \\
(360 \\
\text { questionnaires) }\end{array}$ & $\begin{array}{l}\text { Descriptive statistics } \\
\text { Chi-square tests }\end{array}$ & & $\begin{array}{l}\text { Socio-demographic } \\
\text { Concerns about how food is grown, } \\
\text { preference for IPM-certified corn, } \\
\text { elicited under different marketing } \\
\text { strategies }\end{array}$ \\
\hline Anderson J.C. et al. & 2005 & $\begin{array}{l}\text { Evaluates students' perception of GM and } \\
\text { organic foods and processes }\end{array}$ & $\begin{array}{l}\text { Direct interviews } \\
(340 \\
\text { questionnaires) }\end{array}$ & $\begin{array}{l}\text { Respondents indicated their level of } \\
\text { agreement (8-point Likert scale) }\end{array}$ & & 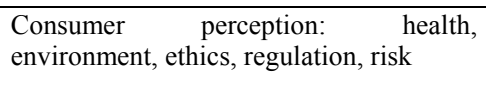 \\
\hline $\begin{array}{l}\text { Baker S., Thompson } \\
\text { K.E. and Engelken } \\
\text { J. }\end{array}$ & 2004 & $\begin{array}{l}\text { Investigates the underlying values driving } \\
\text { food choice behaviour of German and } \\
\text { British consumers }\end{array}$ & $\begin{array}{l}\text { Direct interviews } \\
\text { on a non- } \\
\text { randomly } \\
\text { selected sample } \\
\text { ( } 32 \text { respondents } \\
\text { in each country) }\end{array}$ & $\begin{array}{l}\text { Means-end theory and Laddermap } \\
5.4 \text { software }\end{array}$ & & 25 attributes, 24 consequences, 13 values \\
\hline $\begin{array}{l}\text { Buzby J.C. and } \\
\text { Skees J.R. }\end{array}$ & 1994 & $\begin{array}{l}\text { Reveals the opinion of food shoppers on } \\
\text { food safety in the United States based on } \\
\text { the survey conducted by the University of } \\
\text { Kentucky }\end{array}$ & $\begin{array}{l}\text { National phone } \\
\text { survey, followed } \\
\text { by a mail } \\
\text { questionnaire } \\
\text { (1671 usable) }\end{array}$ & Descriptive statistics & & $\begin{array}{l}\text { Socio-economic (age, gender, race, } \\
\text { income, household size, education), } \\
\text { attitudes about food safety, WTP to } \\
\text { reduce risk }\end{array}$ \\
\hline $\begin{array}{l}\text { Byrne } \\
\text { Toensmeyer } \quad \text { U.J., } \\
\text { German C.L. } \\
\text { Muller H.R. }\end{array}$ & 1992 & $\begin{array}{l}\text { Determines Delmarva consumer attitudes } \\
\text { towards food safety, with an emphasis on } \\
\text { organically grown fresh produce }\end{array}$ & $\begin{array}{l}\text { Mail survey } \\
\text { (1065 usable) }\end{array}$ & $\begin{array}{l}\text { Response types: contingent } \\
\text { valuation on a scale of } 1 \text { to } 7 \text {; } \\
\text { yes, no, or do not know responses; } \\
\text { free choice selection; written } \\
\text { comments; } \\
\text { only descriptive statistics }\end{array}$ & & $\begin{array}{l}\text { Socio-economic (age, gender, income, } \\
\text { education) } \\
\text { Consumer self-ratings of attitudes, } \\
\text { perceptions, and beliefs on various food } \\
\text { safety topics }\end{array}$ \\
\hline Chang Hui Shung & 2004 & $\begin{array}{l}\text { Examines the arguments for and against } \\
\text { the mandatory labelling of organic food in } \\
\text { Australia, compares the political and } \\
\text { marketing environments in which organic } \\
\text { and GMO foods operate, and assesses the } \\
\text { appropriateness of the differing regulatory } \\
\text { responses }\end{array}$ & & Description of labelling schemes & & \\
\hline $\begin{array}{l}\text { Chang Hui Shung } \\
\text { and Zepeda L. }\end{array}$ & 2004 & $\begin{array}{l}\text { Identifies issues that may hinder or } \\
\text { promote demand }\end{array}$ & & Focus group & & $\begin{array}{l}\text { Key research question: who are the } \\
\text { organic consumers? Why do they buy } \\
\text { organic? Are they representative } \\
\text { shoppers or a fringe niche? How do }\end{array}$ \\
\hline
\end{tabular}




\begin{tabular}{|c|c|c|c|c|c|}
\hline & & & & & $\begin{array}{l}\text { consumers feel about the price premiums } \\
\text { generally required for organic products? } \\
\text { How important is certification to } \\
\text { consumer confidence? }\end{array}$ \\
\hline Conner D. & 2004 & $\begin{array}{l}\text { Discusses the options for creating and } \\
\text { maintaining niche markets by providing } \\
\text { information on "process" attributes to } \\
\text { consumers who wish to support a more } \\
\text { sustainable food system, including } \\
\text { labelling alternatives and the role of } \\
\text { government }\end{array}$ & & $\begin{array}{l}\text { Discussion of sustainability and } \\
\text { outlines policy and labeling } \\
\text { mechanisms }\end{array}$ & \\
\hline $\begin{array}{l}\text { Conner D. and } \\
\text { Christy R. }\end{array}$ & 2002 & $\begin{array}{l}\text { Discusses the implications that the } \\
\text { prohibition of the use of the "big } 3 \text { " for } \\
\text { foods labelled organic has on consumers' } \\
\text { WTP, the policy implications of the results } \\
\text { and how firms can use this information for } \\
\text { marketing strategies }\end{array}$ & & CV (open-ended WTP elicitation) & $\begin{array}{l}\text { Socio-economic (age, gender, education, } \\
\text { income) } \\
\text { Attitudes and shopping habits }\end{array}$ \\
\hline $\begin{array}{ll}\text { Eerola } & \text { E.and } \\
\text { Huthala A. } & \end{array}$ & 2005 & $\begin{array}{l}\text { Studies policies designed to encourage } \\
\text { demand for green products under joint } \\
\text { production of a private and public good } \\
\text { using organic food products as example }\end{array}$ & & $\begin{array}{l}\text { The model considers consumers } \\
\text { that differ in income level and } \\
\text { preference for organic products }\end{array}$ & \\
\hline $\begin{array}{l}\text { Fotopolous C. and } \\
\text { Krystallis A. }\end{array}$ & 2002 & $\begin{array}{l}\text { Examines preferences of the Greek } \\
\text { consumer of organic products }\end{array}$ & $\begin{array}{l}\text { Direct interviews } \\
\text { (random } \\
\text { stratified sample, } \\
1612 \\
\text { respondents) }\end{array}$ & Cluster analysis & $\begin{array}{l}\text { Socio-economic (education, income), } \\
\text { food purchase behaviour, media use, diet } \\
\text { habits, attitudes towards the Greek food } \\
\text { origin, attitude towards convenience, } \\
\text { innovative behaviour, attitude towards } \\
\text { advertisement }\end{array}$ \\
\hline $\begin{array}{l}\text { Gifford K. } \quad \text { and } \\
\text { Bernard J.C. }\end{array}$ & 2004 & $\begin{array}{l}\text { Models the price premium of organic food } \\
\text { as a function of the message components } \\
\text { and information provided on the product } \\
\text { packaging. The sub-components of this } \\
\text { goal are to develop a method of recording } \\
\text { and quantifying label claims and messages; }\end{array}$ & & Descriptive statistics & Package type, organic price premium \\
\hline $\begin{array}{l}\text { Govindasamy R., } \\
\text { Italia J. and Liptak } \\
\text { C. }\end{array}$ & 1997 & $\begin{array}{l}\text { Gains a greater insight into the } \\
\text { characteristics and beliefs consumers draw } \\
\text { upon while selecting the produce they } \\
\text { purchase }\end{array}$ & $\begin{array}{l}\text { Mail survey } \\
(656)\end{array}$ & Descriptive statistics & $\begin{array}{l}\text { Nineteen produce characteristics were } \\
\text { ranked by consumers: locally grown } \\
\text { produce and country of origin among the } \\
\text { least important, freshness, taste/flavour, } \\
\text { cleanliness, health value, absence of } \\
\text { pesticides, among the most important } \\
\text { characteristics }\end{array}$ \\
\hline $\begin{array}{ll}\text { Grunert } & \text { S.C.and } \\
\text { Juhl H.J. } & \end{array}$ & 1995 & $\begin{array}{l}\text { Investigates the applicability of the } \\
\text { Schwartz value theory and measurement } \\
\text { approach. }\end{array}$ & $\begin{array}{l}\text { Survey }(174 \\
\text { school teachers })\end{array}$ & $\begin{array}{l}\text { Smallest space analysis, cluster and } \\
\text { discriminant analysis }\end{array}$ & $\begin{array}{l}\text { Socio-economic } \\
56 \text { values } \\
\text { Environmental attitudes }\end{array}$ \\
\hline
\end{tabular}




\begin{tabular}{|c|c|c|c|c|c|}
\hline & & $\begin{array}{l}\text { Relationships between } \\
\text { domains of values and } \\
\text { attitudes are derived }\end{array}$ & & & Buying attitudes towards organic food \\
\hline $\begin{array}{l}\text { Harper G.C. and } \\
\text { Makatouni A. }\end{array}$ & 2002 & $\begin{array}{l}\text { Presents focus group results on consumer } \\
\text { perceptions, attitudes and behaviour in } \\
\text { relation to two key interrelated food trends: } \\
\text { organic food and animal welfare }\end{array}$ & Focus groups & $\begin{array}{l}\text { Discussion of the results from focus } \\
\text { groups }\end{array}$ & $\begin{array}{l}\text { Concerns about health, food safety, } \\
\text { animal welfare, organic and free-range } \\
\text { food }\end{array}$ \\
\hline $\begin{array}{l}\text { Honkanen } \\
\text { Verplanken } \quad \text { B., and } \\
\text { Olsen S.O. }\end{array}$ & 2005 & $\begin{array}{l}\text { Investigates role of ethical motives in } \\
\text { consumers' choice of organic food }\end{array}$ & $\begin{array}{l}\text { Self- } \\
\text { administered } \\
\text { questionnaire } \\
(1283)\end{array}$ & $\begin{array}{l}\text { Descriptive statistics } \\
\text { Confirmatory factor analysis } \\
\text { Structural equation model among } \\
\text { motives, attitudes and intention to } \\
\text { consume organic food }\end{array}$ & $\begin{array}{l}\text { Ethical food choice motives, attitudes } \\
\text { towards consumption of organic food, } \\
\text { choice of organic food }\end{array}$ \\
\hline $\begin{array}{l}\text { Lohr L., Factors } \\
\text { Affecting } \\
\text { International } \\
\text { Demand and Trade } \\
\text { in Organic Food } \\
\text { Products }\end{array}$ & 2000 & $\begin{array}{l}\text { Describes important markets for organic } \\
\text { foods in Europe, Japan, and North America }\end{array}$ & & $\begin{array}{l}\text { Description of the factors affecting } \\
\text { the demand of organic food in } \\
\text { different countries }\end{array}$ & $\begin{array}{l}\text { Factors affecting demand: price } \\
\text { premiums, price-quality trade-off, } \\
\text { country origin of product, GMO content, } \\
\text { social goals }\end{array}$ \\
\hline $\begin{array}{l}\text { Lusk J.L. and } \\
\text { Hudson D. }\end{array}$ & 2004 & $\begin{array}{l}\text { Provides a short introduction to } \\
\text { willingness-to- } \\
\text { pay methodology and provides a discussion } \\
\text { of several different methods used to } \\
\text { estimate } \\
\text { willingness-to-pay }\end{array}$ & & $\begin{array}{l}\text { Discussion of how much of the } \\
\text { work in environmental and } \\
\text { experimental valuation literature } \\
\text { can be extended to agribusiness } \\
\text { applications }\end{array}$ & \\
\hline Makatouni A. & 2002 & $\begin{array}{l}\text { Understands in depth the behavioural } \\
\text { process of parents with respect to organic } \\
\text { food }\end{array}$ & $\begin{array}{l}\text { Laddering } \\
\text { interviews }(40) \\
\text { to parents with } \\
\text { children between } \\
4 \text { and } 12\end{array}$ & Means-end chain theory & $\begin{array}{l}\text { Attributes of organic food } \\
\text { Consequences and personal values linked } \\
\text { to those attributes }\end{array}$ \\
\hline McCluskey J.J. & 2000 & $\begin{array}{l}\text { Models the information asymmetry } \\
\text { problems arising for organic foods in a } \\
\text { game context. The analysis shows that } \\
\text { repeat-purchase relationships and third- } \\
\text { party monitoring are required for high- } \\
\text { quality credence goods to be available }\end{array}$ & & Game theoretic approach & \\
\hline $\begin{array}{l}\text { McGarry Wolf } \text { M., } \\
\text { Johnson } \\
\text { Cochran K. and } \\
\text { Hamilton L. }\end{array}$ & 2002 & $\begin{array}{l}\text { Examines the demographics and category } \\
\text { purchasing behaviour of consumers who } \\
\text { plan to purchase organic lettuce in the } \\
\text { future }\end{array}$ & $\begin{array}{l}\text { Personal } \\
\text { interviews (259) }\end{array}$ & $\begin{array}{l}\text { Comparison of conventional vs } \\
\text { organic. } \\
\text { Variables rated on a } 1-5 \text { desirability } \\
\text { scale }\end{array}$ & $\begin{array}{l}\text { Socio-economic (age, marital status, } \\
\text { gender, education, income, employment } \\
\text { status), consumption, types consumed, } \\
\text { desirability characteristics (including } \\
\text { environmental) }\end{array}$ \\
\hline
\end{tabular}




\begin{tabular}{|c|c|c|c|c|c|c|}
\hline McGarry Wolf M. & 2002 & $\begin{array}{l}\text { Examines the consumer purchase interest } \\
\text { in organic grapes and price sensitivity }\end{array}$ & $\begin{array}{l}\text { Personal } \\
\text { interviews (342) }\end{array}$ & $\begin{array}{l}\text { Laboratory experiment component } \\
\text { of simulated test marketing: } \\
\text { respondents rated nineteen product } \\
\text { characteristics on a five-point } \\
\text { desirability scale to examine the } \\
\text { characteristics of grapes that impact } \\
\text { a consumer's purchase decision. }\end{array}$ & & $\begin{array}{l}\text { Socio-economic (age, marital status, } \\
\text { gender, education, income, employment } \\
\text { status) } \\
\text { Characteristics: freshness, quality, price, } \\
\text { impact on the environment }\end{array}$ \\
\hline $\begin{array}{l}\text { O'Donovan P. and } \\
\text { McCarthy M. }\end{array}$ & 2002 & $\begin{array}{l}\text { Examines consumer demand for organic } \\
\text { meat with special reference to consumers' } \\
\text { perception of food safety }\end{array}$ & $\begin{array}{l}\text { Direct interviews } \\
(250)\end{array}$ & Factor analysis, reliability analysis. & & $\begin{array}{l}\text { Attitudinal statements in relation to } \\
\text { health, environment and animal welfare }\end{array}$ \\
\hline Radman M. & 2005 & $\begin{array}{l}\text { Gains knowledge about consumer attitudes } \\
\text { towards organic products in Croatia }\end{array}$ & $\begin{array}{l}\text { Direct (face-to- } \\
\text { face) interviews } \\
(179)\end{array}$ & $\begin{array}{l}\text { Univariate analysis, chi-square test, } \\
\text { ANOVA and correlation analysis. }\end{array}$ & & $\begin{array}{l}\text { Buying behaviour regarding organic } \\
\text { products, willingness to pay extra price } \\
\text { for these products, knowledge about } \\
\text { supply of organic products in the market, } \\
\text { consumers' satisfaction with supply of } \\
\text { such products, and future intention to buy } \\
\text { ecologically-grown foods }\end{array}$ \\
\hline $\begin{array}{l}\text { Rimal A., Fletcher } \\
\text { S.M., McWatters } \\
\text { K.H., Misra S.K. } \\
\text { and Deodhar S. }\end{array}$ & 2001 & $\begin{array}{l}\text { Examines the relationship between food } \\
\text { safety concerns and the change in food } \\
\text { consumption habits }\end{array}$ & $\begin{array}{ll}\text { Mail } & \text { survey } \\
(236) & \end{array}$ & Regression analysis & $\begin{array}{l}\text { Food safety } \\
\text { concern index } \\
\text { Food } \\
\text { consumption } \\
\text { behaviour index }\end{array}$ & $\begin{array}{l}\text { Socio-economic (health condition, race, } \\
\text { education, employment status, age, } \\
\text { gender) } \\
\text { Attitudes: enforcement of food safety } \\
\text { regulations, use of chemicals, label } \\
\text { Confidence in comments: government } \\
\text { agencies, universities, supermarkets }\end{array}$ \\
\hline $\begin{array}{l}\text { Rimal A. and Moon } \\
\text { W. }\end{array}$ & 2005 & $\begin{array}{l}\text { Examines the role of consumers' perceived } \\
\text { risks and benefits of agro-biotechnology in } \\
\text { shaping the purchase pattern for organic } \\
\text { food among US consumers }\end{array}$ & $\begin{array}{ll}\text { Mail } & \text { survey } \\
(3060) & \end{array}$ & $\begin{array}{l}\text { Frequency analysis, mean test } \\
\text { Ordered probit model }\end{array}$ & $\begin{array}{l}\text { Organic } \\
\text { purchase } \\
\text { frequency }\end{array}$ & $\begin{array}{l}\text { Socio-economic (gender, age, income, } \\
\text { education, race) } \\
\text { Perceived risks and benefits for GM and } \\
\text { organic food, } \\
\text { Food-related attitudes: food safety, price, } \\
\text { trust in government } \\
\text { GM awareness }\end{array}$ \\
\hline Roberts J.A. & 1996 & $\begin{array}{l}\text { Develops a profile of the ecologically } \\
\text { conscious consumer }\end{array}$ & $\begin{array}{l}\text { Nationwide mail } \\
\text { survey (582) }\end{array}$ & $\begin{array}{l}\text { Hierarchical model of multiple } \\
\text { regression }\end{array}$ & $\begin{array}{l}\text { Ecologically } \\
\text { conscious } \\
\text { consumer } \\
\text { behaviour } \\
\text { (ECCB) }\end{array}$ & 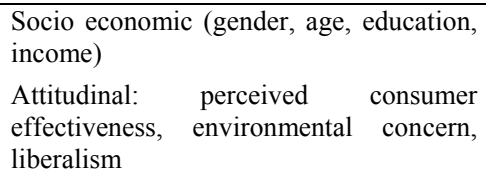 \\
\hline $\begin{array}{l}\text { Robles Robles R., } \\
\text { Vannini L., De la } \\
\text { Puente T. and } \\
\text { Fernandez-Revuelta } \\
\text { J.J. }\end{array}$ & 2005 & $\begin{array}{l}\text { Analyses consumer's attitude versus } \\
\text { organic foods, in order to identify the } \\
\text { factors that influence the demand }\end{array}$ & $\begin{array}{l}\text { Direct interviews } \\
(130)\end{array}$ & Descriptive statistics & & $\begin{array}{l}\text { Factors affecting the purchase of organic } \\
\text { products (quality, health, environment, } \\
\text { guarantee, chemical waste, origin, } \\
\text { scarcity, availability, limited range, } \\
\text { possibility of fraud, lack of knowledge) }\end{array}$ \\
\hline
\end{tabular}




\begin{tabular}{|c|c|c|c|c|c|c|}
\hline & & & & & & attributes of organic products \\
\hline $\begin{array}{l}\text { Tribl C., Salhoefer } \\
\text { K. }\end{array}$ & 2004 & $\begin{array}{l}\text { Addresses the question of how this } \\
\text { environmental benefit can be internalised } \\
\text { best assuming that organic farming creates } \\
\text { a positive externality }\end{array}$ & & $\begin{array}{l}\text { Defines a model to allow the } \\
\text { welfare effects of a subsidy on } \\
\text { organic production and a demand- } \\
\text { side information policy }\end{array}$ & & \\
\hline $\begin{array}{l}\text { Weaver R.D., Evans } \\
\text { D.J. and Luloff A.E. }\end{array}$ & 1992 & $\begin{array}{l}\text { Assesses consumer attitudes towards } \\
\text { pesticide use and residues in fresh produce }\end{array}$ & $\begin{array}{l}\text { Face-to-face } \\
\text { survey }(560)\end{array}$ & Descriptive statistics & & $\begin{array}{l}\text { Characteristics important in purchase } \\
\text { decision, consumer concern for chemical } \\
\text { pesticide use, perceived benefits of } \\
\text { chemical pesticide use, concern in market } \\
\text { intended or actual behaviour, perceptions } \\
\text { of chemical pesticide-free tomatos, } \\
\text { willingness to pay/to purchase pesticide- } \\
\text { free tomatoes. }\end{array}$ \\
\hline $\begin{array}{l}\text { Zepeda L., Douthitt } \\
\text { R. and So-Ye You }\end{array}$ & 2003 & $\begin{array}{l}\text { Presents a model explicitly linking risk } \\
\text { perception (RP) to self-protection actions } \\
\text { (SPAs) and to overall demand for milk }\end{array}$ & $\begin{array}{l}\text { Telephone } \\
\text { survey }(1910)\end{array}$ & $\begin{array}{l}\text { Equations system: two-stage mixed } \\
\text { system }\end{array}$ & $\begin{array}{l}\text { RP } \\
\text { SPA } \\
\text { Milk demand }\end{array}$ & $\begin{array}{l}\text { RP: outrage, attitudinal and demographic } \\
\text { factors } \\
\text { SPA: RP, labelling, economic variables, } \\
\text { demographics, price of SPA } \\
\text { Milk demand:SPA, milk price, price of } \\
\text { SPA, income, demographics }\end{array}$ \\
\hline
\end{tabular}


TABLE B.1.3: Empirical studies: real market data/experimental economics

\begin{tabular}{|c|c|c|c|c|c|c|}
\hline Authors & Year & Purpose of the study & Data & Methodology & $\begin{array}{l}\text { Dependent } \\
\text { variable(s) }\end{array}$ & Explanatory variables \\
\hline $\begin{array}{l}\text { Boland M. and Schroeder } \\
\text { T. }\end{array}$ & 2002 & $\begin{array}{l}\text { Determines the marginal value of attributes to } \\
\text { consumers with respect to natural beef or beef } \\
\text { produced with organic grains }\end{array}$ & $\begin{array}{l}\text { Data for natural organic beef } \\
\text { sales from producer-owned co- } \\
\text { operatives }\end{array}$ & Hedonic price model & $\begin{array}{l}\text { Wholesale primal cut } \\
\text { prices } \\
\text { Carcass revenue }\end{array}$ & 10 product attributes \\
\hline $\begin{array}{l}\text { Galloway K. and Bailey } \\
\text { D.V. }\end{array}$ & 2005 & Determines WTP for "cause" coffee & $\begin{array}{l}\text { Data from experiment (second } \\
\text { price Vickrey style auction) }\end{array}$ & Regression Analysis (104 obs.) & Average WTP bids & $\begin{array}{l}\text { Socio-economic (gender, age, marital } \\
\text { status, children, education) } \\
\text { Coffee characteristics, personal } \\
\text { characteristics (health consciousness, } \\
\text { coffee consumption, life/financial } \\
\text { satisfaction, happiness, donate money, } \\
\text { working hours }\end{array}$ \\
\hline $\begin{array}{l}\text { Gifford K., } \\
\text { Toensmeyer } \\
\text { Bacon R. }\end{array}$ & 2005 & $\begin{array}{l}\text { Determines consumer willingness to pay (WTP) } \\
\text { for non-GM and organic foods relative to } \\
\text { conventional foods and to see if WTP differences } \\
\text { between the three categories vary between fresh } \\
\text { and processed food products }\end{array}$ & $\begin{array}{l}\text { Experimental auctions } \\
\text { (Vickrey's sealed-bid nth-price } \\
\text { auction) with } 133 \text { subjects from } \\
\text { three states }\end{array}$ & Tobit models & WTP bids & $\begin{array}{l}\text { Socio-economic (gender, age, race, } \\
\text { children, education), attitude and } \\
\text { knowledge variables: knowledge of } \\
\text { GM crops, opinion of GM foods, } \\
\text { opinion of organic foods, confidence } \\
\text { in the USDA, label readers, awareness } \\
\text { that GM have been recalled, } \% \text { chance } \\
\text { of GM in the product }\end{array}$ \\
\hline $\begin{array}{l}\text { Glaser L.K. and Thompson } \\
\text { G.D. }\end{array}$ & 1998 & $\begin{array}{l}\text { Models the demand for organic and conventional } \\
\text { frozen vegetables }\end{array}$ & $\begin{array}{l}\text { Supermarket scanner data from } \\
3000 \text { stores (Nielsen) }\end{array}$ & $\begin{array}{l}\text { Almost Ideal Demand System } \\
\text { (AIDS) }\end{array}$ & Consumption shares & Prices, expenditure \\
\hline $\begin{array}{l}\text { Glaser L.K. and Thompson } \\
\text { G.D. }\end{array}$ & 2000 & $\begin{array}{l}\text { Models the demand for organic and conventional } \\
\text { beverage milk }\end{array}$ & $\begin{array}{l}\text { Supermarket scanner data from } \\
3000 \text { stores (Nielsen) } \\
\text { Supermarket scanner data from } \\
13000 \text { stores (IRI) }\end{array}$ & $\begin{array}{l}\text { Almost Ideal Demand System } \\
\text { (AIDS) }\end{array}$ & Consumption shares & Prices, expenditure \\
\hline $\begin{array}{l}\text { Hu Y., Mc Cluskey J.J. } \\
\text { and Durham C.A. }\end{array}$ & 2005 & $\begin{array}{l}\begin{array}{l}\text { Evaluates } \\
\text { apples }\end{array} \\
\text { consumers' WTP for Washington } \\
\end{array}$ & $\begin{array}{l}\text { Consumer tasting survey }(487- \\
\text { Gala apple; } 290-\text { Red } \\
\text { Delicious) }\end{array}$ & $\begin{array}{l}\text { CV: WTP elicited with double } \\
\text { bounded method } \\
\text { ML estimates }\end{array}$ & WTP & $\begin{array}{l}\text { Socio-economic (age education, race) } \\
\text { Purchase frequency } \\
\text { Attributes: firmness, sweetness }\end{array}$ \\
\hline $\begin{array}{l}\text { Lusk J.L., Daniel M.S., } \\
\text { Mark D.R. and Lusk C.L. }\end{array}$ & 2001 & $\begin{array}{l}\text { Explores two important issues in experimental } \\
\text { economics: calibration and auction institution. } \\
\text { Consumer willingness-to-pay bids for corn chips } \\
\text { made with nongenetically modified ingredients are }\end{array}$ & $\begin{array}{l}\text { Auction experiment } \\
\text { First price (18); second price }\end{array}$ & $\begin{array}{l}\text { First- and second-price sealed } \\
\text { bid auctions }\end{array}$ & WTP & $\begin{array}{l}\begin{array}{l}\text { Socio-economic (gender, age, } \\
\text { population) } \\
\text { Consumption, exercise }\end{array}\end{array}$ \\
\hline
\end{tabular}




\begin{tabular}{|c|c|c|c|c|c|c|}
\hline & & elicited in first- and second-price auctions & (32) & Double hurdle model & & $\begin{array}{l}\text { GM: feeling, W. to purchase, concern } \\
\text { Food concerns: cholesterol, bacteria, } \\
\text { hormones }\end{array}$ \\
\hline $\begin{array}{l}\text { Nayga R.M., Tepper B.J. } \\
\text { and Rosenzweig L. }\end{array}$ & 1999 & $\begin{array}{l}\text { Assesses the importance of health/nutrition- } \\
\text { related factors and demographics on food } \\
\text { consumption }\end{array}$ & $\begin{array}{l}1994 \text { Continuing Survey of } \\
\text { Food Intakes by Individuals } \\
\text { (CSFII) and Diet Health } \\
\text { Knowledge Survey (DHKS) } \\
\text { (US Department of Agriculture, } \\
\text { 1996) }\end{array}$ & $\begin{array}{l}\text { Variable preference approach } \\
\text { Seemingly unrelated regression } \\
\text { estimates }\end{array}$ & Average daily intake & $\begin{array}{l}\text { Socio-economic (income, race, age, } \\
\text { residence, gender, employment status, } \\
\text { household size, education, body mass) } \\
\text { health self-perception, overweight, } \\
\text { special diet, pyramid knowledge, diet- } \\
\text { disease knowledge, label use } \\
\text { importance: dietary criteria, nutrition, } \\
\text { price, taste }\end{array}$ \\
\hline Rae A.N. & 2000 & $\begin{array}{l}\text { Measures the importance of several } \\
\text { socioeconomic variables in explaining differences } \\
\text { in household food consumption patterns and } \\
\text { nutrition }\end{array}$ & $\begin{array}{l}1990 \text { National Socio-economic } \\
\text { Survey (Indonesian Central } \\
\text { Bureau of Statistics) }\end{array}$ & $\begin{array}{l}\text { Cluster analysis } \\
\text { Discriminant analysis }\end{array}$ & $\begin{array}{l}\text { Household dietary } \\
\text { pattern }\end{array}$ & $\begin{array}{l}\text { Socio-economic } \\
\text { household size, age, children, } \\
\text { teenagers) }\end{array}$ \\
\hline $\begin{array}{lrr}\text { Roosen J., } & \text { Fox } & \text { J.A., } \\
\text { Hennessy } & \text { D.A. } & \text { and } \\
\text { Schreiber A. } & & \end{array}$ & 1998 & $\begin{array}{l}\text { Measures consumers' willingness to pay (WTP) } \\
\text { for the elimination of one insecticide and also } \\
\text { a whole group of insecticides in apple production }\end{array}$ & $\begin{array}{l}\text { Experimental design (Multiple- } \\
\text { round Vickrey auction) }\end{array}$ & Double hurdle model & $\begin{array}{l}\text { Preference for reduced } \\
\text { pesticide use } \\
\text { WTP for reduced } \\
\text { pesticide use }\end{array}$ & $\begin{array}{l}\text { Socio-economic (gender, age, } \\
\text { education, employment, income, } \\
\text { children, organic shopping, } \\
\text { consumption frequency, apple eaters), } \\
\text { attitude towards policy options on } \\
\text { pesticides, concern about use of } \\
\text { pesticides, food poisoning and food } \\
\text { prices, importance of apple attributes } \\
\text { (flavour, price, damage, pest use) }\end{array}$ \\
\hline $\begin{array}{lll}\text { Thompson } & \text { G.D. } & \text { and } \\
\text { Glaser L.K. } & & \end{array}$ & 2001 & $\begin{array}{l}\text { Analyses the trends in consumption of organic and } \\
\text { conventional baby food during the 1990's }\end{array}$ & $\begin{array}{l}\text { Supermarket scanner data from } \\
3000 \text { stores (Nielsen) } \\
\text { Supermarket scanner data from } \\
13000 \text { stores (IRI) }\end{array}$ & $\begin{array}{l}\text { Quadratic almost ideal demand } \\
\text { system (QUAIDS) }\end{array}$ & Expenditure share & Prices, expenditure \\
\hline $\begin{array}{lll}\text { Thompson } & \text { G.D. } & \text { and } \\
\text { Kidwell J. } & \end{array}$ & 1998 & $\begin{array}{l}\text { Measures actual choice that consumers made in } \\
\text { retail outlets between conventional and organic } \\
\text { produce }\end{array}$ & $\begin{array}{l}\text { Cosmetic defects and prices } \\
\text { collected directly in store. } \\
\text { Consumers socio-economic } \\
\text { traits collected with a } \\
\text { questionnaire (340) }\end{array}$ & $\begin{array}{l}\text { Discrete choice model } \\
\text { Two-equation model }\end{array}$ & $\begin{array}{l}\text { Choice of } \\
\text { organic/conventional; } \\
\text { Choice of store }\end{array}$ & $\begin{array}{l}\text { Socio-economic (income, education, } \\
\text { age, young components, gender, store } \\
\text { distance) } \\
\text { Attributes: defects, price }\end{array}$ \\
\hline von Alvensleben R. & 1998 & Elicits attitudes towards organic food in Germany & 3 surveys $(2000)$ & $\begin{array}{l}\text { Factor analysis } \\
\text { Multiple regression }\end{array}$ & $\begin{array}{l}\text { Consumption index } \\
\text { for organic food }\end{array}$ & Attitudes \\
\hline
\end{tabular}




\begin{tabular}{|c|c|c|c|c|c|c|}
\hline \multirow{3}{*}{$\begin{array}{l}\text { Wier M., Morch Andersen } \\
\text { L., Millock K., O’Doherty } \\
\text { Jensen K. and Rosenqvist } \\
\text { L. }\end{array}$} & \multirow[t]{3}{*}{2005} & \multirow{3}{*}{$\begin{array}{l}\text { Investigates the relationships between Danish } \\
\text { household purchasing behaviour and stated values, } \\
\text { motives and concerns }\end{array}$} & Household panel data (2000) & \multirow{3}{*}{$\begin{array}{l}\text { Almost Ideal Demand System } \\
\text { (AIDS) }\end{array}$} & \multirow{3}{*}{$\begin{array}{l}\text { - Budget share } \\
\text { - Average organic } \\
\text { budget share } \\
\text { - Deviation from the } \\
\text { average organic } \\
\text { budget share }\end{array}$} & \multirow{3}{*}{$\begin{array}{l}\text { Prices, consumer budget } \\
\begin{array}{l}\text { Socio-economic (education, age, } \\
\text { children) }\end{array} \\
\begin{array}{l}\text { Stated values, purchasing barriers } \\
\text { (pesticide health concern, trust) }\end{array}\end{array}$} \\
\hline & & & (GfK Denmark) & & & \\
\hline & & & $\begin{array}{l}\text { Purchase data (attitudes, } \\
\text { perception, values, habits): } \\
\text { survey to the same households } \\
(1609)\end{array}$ & & & \\
\hline $\begin{array}{l}\text { York R. and Hill Gossard } \\
\text { M. }\end{array}$ & 2004 & $\begin{array}{l}\text { Assesses the effects of modernisation and } \\
\text { ecological context on per-capita meat and fish } \\
\text { consumption for } 132 \text { nations }\end{array}$ & Data: UN, World Bank & Trade balance approach & $\begin{array}{l}\text { Per-capita } \\
\text { consumption }\end{array}$ & $\begin{array}{l}\text { Land area per capita, water area p.c., } \\
\text { climate indicator, GDP p.c. }\end{array}$ \\
\hline
\end{tabular}


TABLE B.1.4: Empirical studies: hypothetical markets (Willingness to Pay, conjoint analysis, other models from elicited information)

\begin{tabular}{|c|c|c|c|c|c|c|}
\hline Authors & Year & Main purpose of the study & $\begin{array}{l}\text { Survey method } \\
\text { (usable replies) }\end{array}$ & Methodology & $\begin{array}{l}\text { Dependent } \\
\text { variable(s) }\end{array}$ & Explanatory variables \\
\hline Ara S. & 2003 & $\begin{array}{l}\text { Determines consumers' preferences of multiple } \\
\text { attributes in organic rice }\end{array}$ & Direct interviews (548) & $\begin{array}{l}\text { Conjoint analysis } \\
\text { Logit estimates }\end{array}$ & $\begin{array}{l}\text { Choice among six } \\
\text { experiments }\end{array}$ & $\begin{array}{l}\text { Price, health risk (5-point Likert } \\
\text { scale), environmental quality, } \\
\text { eating quality, organic } \\
\text { certification, fair trade }\end{array}$ \\
\hline Baker G. & 1999 & $\begin{array}{l}\text { Evaluates consumer responses to hypothetical } \\
\text { apple products in a nationwide survey }\end{array}$ & Mail survey (510 usable) & Conjoint analysis & $\begin{array}{l}\text { Choice among } 11 \\
\text { hypothetical products }\end{array}$ & $\begin{array}{l}\text { Price, damage, reduce pesticide } \\
\text { usage, very limited pesticide } \\
\text { usage, certification }\end{array}$ \\
\hline Batte M.T. et al. & 2004 & $\begin{array}{l}\text { Evaluates consumers' WTP for multi-ingredient } \\
\text { processed foods and investigates the willingness } \\
\text { to trade off multi-ingredient foods with varying } \\
\text { levels of organic ingredients }\end{array}$ & Direct interviews (199) & $\begin{array}{l}\begin{array}{l}\text { Contingent Valuation } \\
\text { (payment card) }\end{array} \\
\text { Random Utility Model } \\
\text { multinomial/conditional } \\
\text { logit model estimates }\end{array}$ & $\begin{array}{l}\text { - WTP } \\
\text { - Discrete choice among } \\
\text { four hypothetical multi- } \\
\text { ingredient } \\
\text { products }\end{array}$ & $\begin{array}{l}\text { Socio-economic (age, income, } \\
\text { children, education, health index, } \\
\text { race, gender), National Organic } \\
\text { Program (NOP) awareness }\end{array}$ \\
\hline Boccaletti S. and Nardella M. & 2000 & $\begin{array}{l}\text { Evaluates the potential for organic produce, in } \\
\text { particular if } \\
\text { consumers would be willing to pay price } \\
\text { premiums }\end{array}$ & $\begin{array}{l}\text { Direct interviews (400 } \\
\text { usable) }\end{array}$ & $\begin{array}{l}\begin{array}{l}\text { Contingent } \\
\text { (payment card) }\end{array} \\
\text { Multinomial logit }\end{array}$ & WTP & $\begin{array}{l}\text { Socio-economic (age, gender, } \\
\text { education, urban, children, } \\
\text { income) } \\
\text { Knowledge of pesticide-borne } \\
\text { risks } \\
\text { Pesticide concern index, food } \\
\text { borne risk concern index }\end{array}$ \\
\hline $\begin{array}{l}\text { Buzby J.C., Ready R.C. and } \\
\text { Skees J.R. }\end{array}$ & 1995 & $\begin{array}{l}\text { Evaluates costs and benefits from banning a } \\
\text { specific post-harvest pesticide }\end{array}$ & Mail survey (548 usable) & $\begin{array}{l}\text { Contingent Valuation } \\
\text { (payment card) } \\
\text { Regression analysis }\end{array}$ & WTP & $\begin{array}{l}\text { Socio-economic (gender, } \\
\text { income, age, education, number } \\
\text { of shoppers in the household), } \\
\text { two attitudinal factors about } \\
\text { safety of current pesticide use } \\
\text { and total ban }\end{array}$ \\
\hline $\begin{array}{l}\text { Byrne P.J., Toensmeyer U.C., } \\
\text { German C.L. and Muller H.R. }\end{array}$ & 1991 & $\begin{array}{l}\text { Determines which consumers are and are not } \\
\text { buying organic produce, and analyses their } \\
\text { characteristic relationships between organic and } \\
\text { conventionally grown produce, as well as their } \\
\text { purchase likelihoods }\end{array}$ & Mail survey (753 usable) & $\begin{array}{l}\text { Multinomial logit } \\
\text { Ordered logit }\end{array}$ & $\begin{array}{l}\text { - Organic produce } \\
\text { purchase experience } \\
\text { - Rating of organic vs. } \\
\text { conventional produce }\end{array}$ & $\begin{array}{l}\text { Socio-economic (age, gender, } \\
\text { income, education), rating of } \\
\text { organic produce compared to } \\
\text { conventional, perception of } \\
\text { organic costs compared to } \\
\text { conventional }\end{array}$ \\
\hline Chakraborty K. & 2005 & $\begin{array}{l}\text { Measures consumers' WTP for Recombinant } \\
\text { Bovine Somatotropin (BST) -free milk and to }\end{array}$ & Mail survey (700 usable) & $\begin{array}{l}\text { Contingent Valuation } \\
\text { Probit }\end{array}$ & $\begin{array}{l}\text { I would/I would not be } \\
\text { willing to pay a premium } \\
\text { for BST-free milk }\end{array}$ & $\begin{array}{l}\text { Socio-economic } \text { (gender, } \\
\text { income, age, education, children, } \\
\text { household size), support of }\end{array}$ \\
\hline
\end{tabular}




\begin{tabular}{|c|c|c|c|c|c|c|}
\hline & & explore the factors influencing it & & & & $\begin{array}{l}\text { mandatory } r \text { labelling, } \\
\text { information, health risk, } \\
\text { mandatory BST label, milk } \\
\text { consumption }\end{array}$ \\
\hline $\begin{array}{l}\text { Cicia G., Del Giudice T. and } \\
\text { Scarpa R. }\end{array}$ & 2002 & $\begin{array}{l}\text { Investigates the preferences of regular consumers } \\
\text { of organic food allowing for preference } \\
\text { heterogeneity }\end{array}$ & Direct interviews (198) & $\begin{array}{l}\text { Complete ranking and } \\
\text { random utility }\end{array}$ & $\begin{array}{l}\text { Eight choices to rank- } \\
\text { order nine product } \\
\text { profiles }\end{array}$ & $\begin{array}{l}\text { Product attributes included price, } \\
\text { origin of production, type of } \\
\text { certification and visual } \\
\text { appearance }\end{array}$ \\
\hline Corsi A. & 2005 & $\begin{array}{l}\text { Assesses consumers habits concerning beef } \\
\text { consumption and their responses to the BSE both } \\
\text { immediately and in the longer term; consumers' } \\
\text { attitudes and willingness to pay for organic beef }\end{array}$ & $\begin{array}{l}\text { Two random telephone } \\
\text { surveys }(402+330)\end{array}$ & $\begin{array}{l}\text { Probit } \\
\text { Parametric estimates }\end{array}$ & $\begin{array}{l}\text { Maintain } \begin{array}{r}\text { beef } \\
\text { consumption } \\
\text { change }\end{array} \\
\text { after BSE } \\
\text { Difference in expenditure }\end{array}$ & $\begin{array}{l}\text { Socio-.economic (age, } \\
\text { education, household size, town } \\
\text { size, gender, income), familiar } \\
\text { with organic products }\end{array}$ \\
\hline $\begin{array}{l}\text { Cranfield J.A.L. and Magnusson } \\
\text { E. }\end{array}$ & 2003 & $\begin{array}{l}\text { Determines if Canadian consumers would pay a } \\
\text { premium for pesticide-free food products }\end{array}$ & Mail survey (295 usable) & $\begin{array}{ll}\text { Contingent } & \text { Valuation } \\
\text { (payment card) } & \\
\text { Ordered probit } & \end{array}$ & WTP & $\begin{array}{l}\text { Socio-economic (residence, } \\
\text { children, age, education, income) } \\
\text { Try new products, shopping at } \\
\text { health food stores, pesticide } \\
\text { scale, sustainability scale, heard } \\
\text { of pesticide-free production }\end{array}$ \\
\hline Dunlap R.E. and Beus C.E. & 1992 & $\begin{array}{l}\text { Investigates the public's view of pesticides use. } \\
\text { Individual attitudes towards several pesticide } \\
\text { issues are analysed. }\end{array}$ & Telephone survey (437) & $\begin{array}{l}\text { Percentage distributions } \\
\text { Principal components } \\
\text { analysis regression }\end{array}$ & $\begin{array}{l}\text { Necessity of pesticide } \\
\text { use } \\
\text { Safety of pesticide use } \\
\text { Trust in food industry for } \\
\text { pesticides use }\end{array}$ & $\begin{array}{l}\text { Socio-economic (gender, age, } \\
\text { education, income, residence) }\end{array}$ \\
\hline Durham C.A. and Andrade D. & 2005 & $\begin{array}{l}\text { Evaluates the relative strength of health and } \\
\text { environmental motivations in the purchase of } \\
\text { organic foods }\end{array}$ & Direct interviews (257) & $\begin{array}{l}\text { Discrete choice model } \\
\text { (random utility) }\end{array}$ & $\begin{array}{l}\text { Prefer organic } \\
\text { High/low buying }\end{array}$ & $\begin{array}{l}\text { Socio-economic (age, income } \\
\text { education, children, gender) } \\
\text { Environmental purchasing } \\
\text { behaviour } \\
\text { Energy conservation and } \\
\text { recycling behaviour } \\
\text { Nutrition, price, appearance } \\
\text { Health environmental sensitivity } \\
\text { Fitness, personal health } \\
\text { responsibility }\end{array}$ \\
\hline Eom Y.S. & 1994 & $\begin{array}{l}\text { Develops a theoretical model integrating } \\
\text { consumer-stated behaviour with risk perception } \\
\text { about pesticide residues }\end{array}$ & $\begin{array}{l}\text { Questionnaires } \\
\text { distributed by trained } \\
\text { interviewers with self- } \\
\text { addressed envelope } \\
(567)\end{array}$ & $\begin{array}{l}\text { Random utility model } \\
\text { Probit estimates }\end{array}$ & $\begin{array}{l}\text { Intention to purchase } \\
\text { safer produce }\end{array}$ & $\begin{array}{l}\text { Socio-economic (income, age, } \\
\text { education, gender, household } \\
\text { size, children) } \\
\text { price increase, risk reduction } \\
\text { between the } 2 \text { product types, } \\
\text { health risk, health consciousness. }\end{array}$ \\
\hline
\end{tabular}




\begin{tabular}{|c|c|c|c|c|c|c|}
\hline $\begin{array}{l}\text { Gil J. M, Gracia A. and Sanchez } \\
\text { M. }\end{array}$ & 2000 & $\begin{array}{l}\text { Analyses consumer's WTP for organic food, with } \\
\text { consumers segmented according to lifestyles }\end{array}$ & Direct interviews (400) & 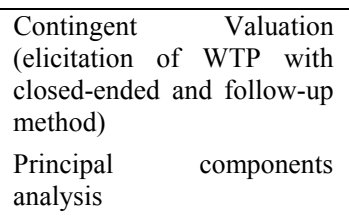 & & $\begin{array}{l}\text { socio-economic (age, gender, } \\
\text { education level, household size, } \\
\text { income), consumption level, } \\
\text { lifestyles, environmental } \\
\text { attitudes (conservation, concern), } \\
\text { organic products attitudes }\end{array}$ \\
\hline Govindasamy R. and Italia J. & 1997 & $\begin{array}{l}\text { Evaluates which demographic characteristics } \\
\text { cause consumers to be more likely to purchase } \\
\text { IPM grown produce }\end{array}$ & $\begin{array}{l}\text { Direct consumer survey } \\
\text { distributed at } 5 \text { grocery } \\
\text { retailers and collected by } \\
\text { mail ( } 291 \text { responses) }\end{array}$ & Logit model & $\begin{array}{l}\text {-Purchase only } \\
\text { conventional } \\
\text {-Heard of IPM } \\
\text {-Willingness to purchase } \\
\text { IPM } \\
\text {-WTP }>10 \% \text { for IPM } \\
\text {-WTP }>10 \% \text { for organic }\end{array}$ & $\begin{array}{l}\text { Socio-economic (age, gender, } \\
\text { education level, household size, } \\
\text { income, marital status, urban, } \\
\text { primary food purchaser) } \\
\text { heard of IPM, pesticide risk, } \\
\text { negative environmental impact } \\
\text { of pesticides, use farmers' } \\
\text { market, own garden grown } \\
\text { produce, use of media for } \\
\text { information. }\end{array}$ \\
\hline Govindasamy R. and Italia J. & 1998 & $\begin{array}{l}\text { Empirically evaluates consumer concern about } \\
\text { pesticide residues and analyses the effect of } \\
\text { socio-demographic factors on pesticide residue } \\
\text { concern. }\end{array}$ & $\begin{array}{l}\text { Mail survey }(1195,656 \\
\text { responses) }\end{array}$ & Logit model & $\begin{array}{ll}\text { Adversion } & \text { towards } \\
\text { pesticide usage }\end{array}$ & $\begin{array}{l}\text { Socio-economic (age, gender, } \\
\text { education, income, children, } \\
\text { marital status, urban, frequent } \\
\text { supermarket visitor, household } \\
\text { size) }\end{array}$ \\
\hline Govindasamy R. and Italia J. & 1999 & $\begin{array}{l}\text { Evaluates which demographic characteristics } \\
\text { affect consumers' WTP for organic produce }\end{array}$ & $\begin{array}{l}\text { Direct consumer survey } \\
\text { distributed at } 5 \text { grocery } \\
\text { retailers and collected by } \\
\text { mail(291 responses) }\end{array}$ & Logit model & $\begin{array}{l}\text { Pay a premium for } \\
\text { organic }\end{array}$ & $\begin{array}{l}\text { Socio-economic (age, gender, } \\
\text { education level, household size, } \\
\text { income), use farmers' market, } \\
\text { purchase organic, heard of } \\
\text { organic, pesticide risk, own } \\
\text { garden grown produce, use of } \\
\text { media, primary food purchaser, } \\
\text { try new foods, negative } \\
\text { environmental impact of } \\
\text { pesticides, shop in many food } \\
\text { stores }\end{array}$ \\
\hline $\begin{array}{l}\text { Govindasamy R., Italia J. and } \\
\text { Adelaja A. }\end{array}$ & 2001 & $\begin{array}{l}\text { Evaluates the demographic characteristics that } \\
\text { influence consumers to pay a premium for 1PM } \\
\text { grown produce }\end{array}$ & $\begin{array}{l}\text { Direct consumer survey } \\
\text { distributed at } 5 \text { grocery } \\
\text { retailers and collected by } \\
\text { mail ( } 291 \text { responses) }\end{array}$ & Logit model & Pay a premium for IPM & $\begin{array}{l}\text { Socio-economic (age, gender, } \\
\text { education level, household size, } \\
\text { income, marital status, urban, } \\
\text { primary food purchaser) } \\
\text { heard of IPM, pesticide risk, } \\
\text { negative environmental impact } \\
\text { of pesticides, use farmers' } \\
\text { market, own garden grown } \\
\text { produce, use of media for } \\
\text { information, shop in many food } \\
\text { stores }\end{array}$ \\
\hline
\end{tabular}




\begin{tabular}{|c|c|c|c|c|c|c|}
\hline $\begin{array}{l}\text { Groff A.J., Kreider C.R. and } \\
\text { Toensmeyer U.C. }\end{array}$ & 1993 & $\begin{array}{l}\text { Determines the overall probabilities and marginal } \\
\text { demographic effects for consumer rating of } \\
\text { organics versus conventional produce, and } \\
\text { the importance of various factors in consumer } \\
\text { organic produce purchasing decisions }\end{array}$ & $\begin{array}{lr}\text { Mail survey } & (6155 \\
\text { households, } & \text { response } \\
\text { rate } 12.2 \%) & \end{array}$ & Ordered logit models & $\begin{array}{l}\text { Rating of organics vs. } \\
\text { conventional } \\
\text { Rating of the importance } \\
\text { of where produce was } \\
\text { grown } \\
\text { Rating of the importance } \\
\text { of brand name }\end{array}$ & $\begin{array}{l}\text { Socio-economic (age, gender, } \\
\text { education, children, marital } \\
\text { status, income) }\end{array}$ \\
\hline $\begin{array}{l}\text { Hansmann R., Scholz R.W., } \\
\text { Francke C-J. A.C. and Weymann } \\
\text { M. }\end{array}$ & 2005 & $\begin{array}{l}\text { Assesses environmental and economic } \\
\text { consequences of food consumption }\end{array}$ & $\begin{array}{l}215 \text { pupils in } 6 \text { classes } \\
\text { played the game }\end{array}$ & $\begin{array}{ll}\text { SIMULME } \\
\text { game) }\end{array}$ & & $\begin{array}{l}\text { Economic (unemployment } \\
\text { figures, the number of persons } \\
\text { employed } \\
\text { in agriculture, the average } \\
\text { income of farms, and the } \\
\text { development of consumers' } \\
\text { purchasing } \\
\text { power), ecological (the size of } \\
\text { the area occupied by agriculture } \\
\text { in Switzerland, the ecological } \\
\text { state of the agricultural area, the } \\
\text { implementation of fair and } \\
\text { humane animal husbandry, and } \\
\text { the global ecological situation) }\end{array}$ \\
\hline Hearne R.R. and Volcan M.M. & 2002 & $\begin{array}{l}\text { Elicits Costa Rican consumer preferences for } \\
\text { different attributes of organic and conventional } \\
\text { vegetables in a hypothetical market }\end{array}$ & Direct interviews (432) & $\begin{array}{l}\text { Discrete choice model } \\
\text { Logit and conditional logit } \\
\text { models }\end{array}$ & Product alternatives & $\begin{array}{l}\text { Blue label, blue and green label, } \\
\text { size, appearance, price } \\
\text { (demographics in a different } \\
\text { model) }\end{array}$ \\
\hline Huang C.L. & 1996 & $\begin{array}{l}\text { Analyses simultaneously consumers' preferences } \\
\text { and attitudes towards organic produce }\end{array}$ & $\begin{array}{l}\text { Mail survey }(580,381 \\
\text { completed) }\end{array}$ & $\begin{array}{l}\text { Discrete choice model } \\
\text { Maximum } \quad \text { Likelihood } \\
\text { estimates }\end{array}$ & $\begin{array}{l}\text { Prefer to buy organic } \\
\text { fresh produce } \\
\text { Would buy organic even } \\
\text { with sensory defects }\end{array}$ & $\begin{array}{l}\text { Socio-economic (gender, age } \\
\text { education, household size, } \\
\text { income, race) } \\
\text { attitudes: pesticides concern, } \\
\text { pesticide ban, certification, } \\
\text { nutritional value, appearance, } \\
\text { low price }\end{array}$ \\
\hline James S., Burton M. & 2003 & $\begin{array}{l}\text { Examines the conditions under which consumers } \\
\text { would purchase GM foods }\end{array}$ & $\begin{array}{l}\text { Mail survey }(2080,370 \\
\text { responses) }\end{array}$ & $\begin{array}{l}\text { Discrete choice model } \\
\text { Conditional logit model }\end{array}$ & Product alternatives & $\begin{array}{l}\text { Socio-economic (age, education, } \\
\text { income, mean shoping bill, } \\
\text { organic food buying habits, } \\
\text { occupation, heard of GM, } \\
\text { children) } \\
\begin{array}{l}\text { Attributes: weekly food bill, } \\
\text { production }\end{array}\end{array}$ \\
\hline
\end{tabular}




\begin{tabular}{|c|c|c|c|c|c|c|}
\hline & & & & & & $\begin{array}{l}\text { (conventional, GM), level of on- } \\
\text { farm chemical use, } \\
\text { environmental risk, health risk }\end{array}$ \\
\hline Jolly D.A. & 1991 & $\begin{array}{l}\text { Elicitation of attitudinal and perceptual responses } \\
\text { to questions about food safety concerns; the } \\
\text { quality of conventional vs organic produce; and } \\
\text { purchasing patterns of organic products. }\end{array}$ & Mail survey (1769) & Analysis of variance & & $\begin{array}{l}\text { Socio economic (occupation, } \\
\text { age, size of community) } \\
\text { Attitudinal factors (levels of } \\
\text { concern for chemical residues, } \\
\text { additives and preservatives, } \\
\text { artificial coloring, radiation } \\
\text { byproducts, salt and sugar). }\end{array}$ \\
\hline $\begin{array}{l}\text { Larue B., West G.E., Gendron C. } \\
\text { and Lambert R. }\end{array}$ & 2004 & $\begin{array}{l}\text { Characterises Canadian consumer response to } \\
\text { GM functional food vs. conventional or organic } \\
\text { food with the same functional property }\end{array}$ & Telephone survey (383) & Discrete choice model & $\begin{array}{l}\text { Product alternatives } \\
\text { (conventional, organic, } \\
\text { GM) }\end{array}$ & $\begin{array}{l}\text { Attributes: price, GM, organic, } \\
\text { health property, health property } \\
\text { (organic), health property (GM). }\end{array}$ \\
\hline Lohr L. and Semali A. & 2000 & $\begin{array}{l}\text { Identifies significant factors in the retail decision } \\
\text { to sell organic foods and quantifies the effects of } \\
\text { retailer attitudes on behaviour }\end{array}$ & $\begin{array}{l}\text { Direct interviews } \\
\text { store managers }(90)\end{array}$ & Probit model & Sell organic food & $\begin{array}{l}\text { Customers characteristics, chain } \\
\text { type, sales/sq.feet, manager's } \\
\text { education, support for organic, } \\
\text { competitive and economic } \\
\text { factors, information, price } \\
\text { premium, margin }\end{array}$ \\
\hline Loureiro M.L. and Hine S. & 2002 & $\begin{array}{l}\text { Elicits consumers' willingness to pay (WTP) for a } \\
\text { labelled value-added potato that could be } \\
\text { marketed as organic, GMO-free, or Colorado } \\
\text { grown (local) }\end{array}$ & Direct interviews (437) & $\begin{array}{l}\text { Contingent Valuation: WTP } \\
\text { elicitation with payment } \\
\text { card }\end{array}$ & WTP & $\begin{array}{l}\text { Socio-economic } \text { (gender, } \\
\text { children, income, age, education, } \\
\text { upper class), importance of } \\
\text { nutrition, importance of } \\
\text { freshness }\end{array}$ \\
\hline Loureiro M.L.and Lotade J. & 2005 & $\begin{array}{l}\text { Reveals consumer preferences for ethical and } \\
\text { environmentally sound labelling programmes in } \\
\text { coffee }\end{array}$ & Direct interviews (284) & $\begin{array}{l}\text { Contingent Valuation: WTP } \\
\text { elicitation with payment } \\
\text { card } \\
\text { Weibull model }\end{array}$ & WTP & $\begin{array}{l}\text { Socio-economic (education, } \\
\text { income, age, gender), concerns: } \\
\text { welfare, environment }\end{array}$ \\
\hline $\begin{array}{l}\text { Loureiro M.L., McCluskey J.J. } \\
\text { and Mittelhammer R.C. }\end{array}$ & 2001 & $\begin{array}{l}\text { Assesses consumer choice of eco-labelled, } \\
\text { organic, and regular apples, and identifies socio- } \\
\text { demographic characteristics affecting the choice } \\
\text { among those three alternatives. Estimates based } \\
\text { on stated preferences are compared with actual } \\
\text { purchase behaviour obtained from a market } \\
\text { experiment }\end{array}$ & Direct interviews (285) & $\begin{array}{l}\text { Discrete choice model } \\
\text { Multinomial logit }\end{array}$ & $\begin{array}{l}\text { Product alternatives } \\
\text { (organic, eco-labelled, } \\
\text { regular apples) }\end{array}$ & $\begin{array}{l}\text { Socio-economic (education, } \\
\text { income, age, gender, children, } \\
\text { household size, race, main } \\
\text { shopper) } \\
\text { Concerns: environment, food } \\
\text { safety, quality, quality of eco- } \\
\text { labelled apples }\end{array}$ \\
\hline $\begin{array}{l}\text { Loureiro M.L., McCluskey J.J. } \\
\text { and Mittelhammer R.C. }\end{array}$ & 1999 & $\begin{array}{l}\text { Assesses consumer preferences for sustainable } \\
\text { agricultural, organic, and conventional apples. } \\
\text { Estimates based on stated preferences are } \\
\text { compared with actual purchase behaviour } \\
\text { obtained from a market experiment }\end{array}$ & $\begin{array}{l}\text { Direct interviews (289) } \\
\text { Scanner data }\end{array}$ & $\begin{array}{l}\text { Discrete choice model } \\
\text { Multinomial logit } \\
\text { Hedonic price analysis }\end{array}$ & $\begin{array}{l}\text { Product alternatives } \\
\text { (organic, eco-labelled, } \\
\text { regular apples) }\end{array}$ & $\begin{array}{l}\text { Socio-economic (education, } \\
\text { income, age, gender, children, } \\
\text { household size, race, main } \\
\text { shopper) } \\
\text { Concerns: environment, food } \\
\text { safety, quality, quality of eco- }\end{array}$ \\
\hline
\end{tabular}




\begin{tabular}{|c|c|c|c|c|c|c|}
\hline & & & & & & labelled apples \\
\hline Lusk J.L. and Coble K.H. & 2005 & $\begin{array}{l}\text { Investigates the effect of risk preferences and risk } \\
\text { perceptions on acceptance of GM food }\end{array}$ & $\begin{array}{l}\text { Direct interviews }(50 \\
\text { undergraduate students) }\end{array}$ & $\begin{array}{lr}\begin{array}{l}\text { Nonhypothetical } \\
\text { preference }\end{array} & \begin{array}{r}\text { risk- } \\
\text { elicitation }\end{array} \\
\text { experiment. } & \\
\text { Probit model } & \end{array}$ & $\begin{array}{l}\text { Eat GM food } \\
\text { Purchase GM food }\end{array}$ & $\begin{array}{l}\text { Socio-economic characteristics } \\
\text { of interviewed students (race, } \\
\text { loan, income, work, age, gender, } \\
\text { freshman) }\end{array}$ \\
\hline $\begin{array}{l}\text { Magnusson E. and Cranfield } \\
\text { A.L. }\end{array}$ & 2005 & $\begin{array}{l}\text { Assesses what food products consumers would } \\
\text { purchase if available in a pesticide-free } \\
\text { production form and what factors affect demand } \\
\text { for PFP food products }\end{array}$ & $\begin{array}{l}\text { Mail survey }(2000,320 \\
\text { usable) }\end{array}$ & $\begin{array}{l}\text { Discrete choice model } \\
\text { Probit model }\end{array}$ & Purchase PFP & $\begin{array}{l}\text { Socio-economic (residence, } \\
\text { income, education, age, gender, } \\
\text { children, marital status) } \\
\text { Try new food, health food stores } \\
\text { Concerns: pesticide residues, } \\
\text { pesticides in the environment, } \\
\text { labels } \\
\text { Heard of PFP, switch grocery } \\
\text { stores to find PFP, WTP for PFP }\end{array}$ \\
\hline $\begin{array}{l}\text { Misra S., Huang C.L. and Ott } \\
\text { S.L. }\end{array}$ & 1991 & $\begin{array}{l}\text { Analyses consumer preferences for testing and } \\
\text { certification of fresh produce and consumers' } \\
\text { willingness to pay for fresh produce that is } \\
\text { certified as free of pesticide residues (FPR) }\end{array}$ & $\begin{array}{l}\text { Mail survey }(580,389 \\
\text { returned) }\end{array}$ & $\begin{array}{l}\text { CV: WTP elicitation with } \\
\text { checklist } \\
\text { Ordered probit model }\end{array}$ & WTP & $\begin{array}{l}\text { Socio-economic (residence, } \\
\text { income, education, age, gender, } \\
\text { race), expectation of financial } \\
\text { status, importance of testing and } \\
\text { certification, pesticides concern } \\
\text { index }\end{array}$ \\
\hline $\begin{array}{l}\text { Nganje E.W., Kaitibie S. and } \\
\text { Taban T. }\end{array}$ & 2005 & $\begin{array}{l}\text { Evaluates the determinants of food safety risk } \\
\text { perception gaps }\end{array}$ & Direct interviews (363) & Discrete choice model & $\begin{array}{l}\text { Consumer risk } \\
\text { perception, } \\
\text { consumption of bison } \\
\text { at/away from home }\end{array}$ & $\begin{array}{l}\text { Socio-economic (residence, } \\
\text { gender, age, income) } \\
\text { Personal health influence, } \\
\text { perceived locus of control, } \\
\text { consumption } \\
\text { outrage/awareness: food safety } \\
\text { risk, safe handling, TV info, } \\
\text { magazines, labels }\end{array}$ \\
\hline $\begin{array}{l}\text { Roheim C.A., Johnston R.J., } \\
\text { Greer J. and Donath H. }\end{array}$ & 2005 & $\begin{array}{l}\text { Assesses trade-offs between species, price, and } \\
\text { the presence of ecolabels for popular fresh } \\
\text { seafood species }\end{array}$ & $\begin{array}{l}\text { Mail survey }(1414,368 \\
\text { usable) }\end{array}$ & $\begin{array}{l}\text { Conjoint analysis } \\
\text { Random utility (discrete } \\
\text { choice) }\end{array}$ & Product alternatives & $\begin{array}{l}\text { Socio-economic (age, household } \\
\text { size, income, seafood } \\
\text { expenditure, consumption } \\
\text { frequency, feel overfishing, } \\
\text { member of environmental group) } \\
\text { Product attributes (price, label, } \\
\text { fish type) }\end{array}$ \\
\hline $\begin{array}{l}\text { Scarpa R., Spalatro F. and } \\
\text { Canavari M. }\end{array}$ & 2003 & $\begin{array}{l}\text { Investigates preferences for potatoes obtained } \\
\text { from different environment-friendly production } \\
\text { methods }\end{array}$ & $\begin{array}{l}\text { Stated preference data } \\
(2000 \text { households) using } \\
\text { Nielsen } \quad \text { telematic } \\
\text { network }\end{array}$ & Mixed logit & Product alternatives & $\begin{array}{l}\text { Attributes: organic, Integrated } \\
\text { Crop Management, quality } \\
\text { certification, size, white pulp, } \\
\text { appearance, bag, domestic }\end{array}$ \\
\hline
\end{tabular}




\begin{tabular}{|c|c|c|c|c|c|c|}
\hline & & & & & & origin, price \\
\hline $\begin{array}{l}\text { Shuzzler A., Govindasamy R. } \\
\text { and Adelaja A. }\end{array}$ & 2003 & $\begin{array}{l}\text { Documents the characteristics of frequent buyers } \\
\text { of organic food and the characteristics of those } \\
\text { who are willing to pay } 10 \% \text { or more for organic } \\
\text { food }\end{array}$ & $\begin{array}{lrr}\text { Data } & \text { extracted } & \text { from } \\
\text { different surveys } & \end{array}$ & Logit model & $\begin{array}{l}\text { Frequently buy organic } \\
\text { Willing to pay } \geq 10 \%\end{array}$ & $\begin{array}{l}\text { Socio-economic (household size, } \\
\text { residence, gender, age, } \\
\text { education, income, marital } \\
\text { status) } \\
\text { Label concern, farmers market, } \\
\text { advertis use, absence of } \\
\text { pesticide, switch supermarket, } \\
\text { higher quality, higher variety, } \\
\text { pesticide risk, fertilizer risk, } \\
\text { know IPM, quality affects } \\
\text { shopping. }\end{array}$ \\
\hline $\begin{array}{l}\text { Soregaroli C., Boccaletti S. and } \\
\text { Moro D. }\end{array}$ & 2003 & $\begin{array}{l}\text { Assesses consumers' attitude towards foods } \\
\text { obtained from the application of biotechnologies } \\
\text { and foods labelled as "GM-free" and measures } \\
\text { WTP for GM-free and WTA for GM food }\end{array}$ & $\begin{array}{l}\text { Direct interviews (500, } \\
459 \text { usable) }\end{array}$ & $\begin{array}{l}\text { CV: WTP/WTA elicited } \\
\text { with payment card } \\
\text { Binomial probit } \\
\text { Ordered probit }\end{array}$ & $\begin{array}{l}\text { WTP (premium for GM- } \\
\text { free food) } \\
\text { WTA (discount for GM } \\
\text { food) }\end{array}$ & $\begin{array}{l}\text { Socio-economic (gender, age, } \\
\text { household size, children, } \\
\text { education, income, employment } \\
\text { status, residence, usual shopper) } \\
\text { Awareness and knowledge of } \\
\text { GMO, confidence in GMO-free } \\
\text { label } \\
\text { Attitudes towards GMO: risk, } \\
\text { trust, useful, agree }\end{array}$ \\
\hline Underhill S.E. and Figueroa E.E. & 1996 & $\begin{array}{l}\text { Examinees the potential for marketing fresh fruits } \\
\text { and vegetables with labels indicating } \\
\text { enhanced food and/or environmental safety } \\
\text { attributes as compared to conventional produce }\end{array}$ & $\begin{array}{l}\text { Mail survey }(1500,534 \\
\text { usable) }\end{array}$ & Ordered logit & $\begin{array}{l}\text { Likelihood of purchase } \\
(1-5 \text { scale) for organic, } \\
\text { certified } \\
\text { certified organic, } \\
\text { residue-free label, IPM } \\
\text { label, } \\
\begin{array}{l}\text { WTP (for the same } \\
\text { variants) }\end{array}\end{array}$ & $\begin{array}{l}\text { Socio-economic (age, urban, } \\
\text { gender, income), knowledge }\end{array}$ \\
\hline Veeman M. and Adamowicz W. & 2000 & $\begin{array}{l}\text { Assesses Alberta consumers' stated preferences } \\
\text { and purchase behaviour for foods exhibiting a } \\
\text { range of environmental risks; response of a } \\
\text { random sample of consumers to the use of } \\
\text { recombinant bovine } \\
\text { somatotrophin (rBST) in milk production }\end{array}$ & $\begin{array}{l}\text { Telephone survey for } \\
\text { preferences towards food } \\
\text { with environmental risk } \\
\text { (1240) } \\
\text { Mail survey for response } \\
\text { to the use of rBST (400, } \\
294 \text { returned) }\end{array}$ & $\begin{array}{l}\text { Discrete choice model } \\
\text { Multinomial logit models }\end{array}$ & $\begin{array}{l}\text { Choice to restrict } \\
\text { pesticide/hormone use } \\
\text { Milk alternatives }\end{array}$ & $\begin{array}{l}\text { Socio-economic (age, gender, } \\
\text { children, income, education), } \\
\text { rBST knowledge, milk type, } \\
\text { rBST presence, price }\end{array}$ \\
\hline Verhoef P.C. & 2005 & $\begin{array}{l}\text { Investigatees the impact of economic and } \\
\text { marketing variables, emotions, social norms, and } \\
\text { environment-related variables on Dutch } \\
\text { consumers' preference for, and purchase }\end{array}$ & $\begin{array}{l}\text { Mail survey }(309,269 \\
\text { usable) }\end{array}$ & $\begin{array}{l}\text { Multinomial ordered probit } \\
\text { Binary probit }\end{array}$ & 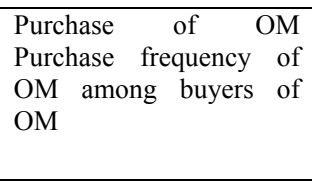 & $\begin{array}{l}\text { Socio-economic (age, household } \\
\text { size, income, education, gender) } \\
\text { Quality perception OM, price } \\
\text { perception OM, distribution } \\
\text { perception, fear, guilt empathy, }\end{array}$ \\
\hline
\end{tabular}




\begin{tabular}{|c|c|c|c|c|c|c|}
\hline & & frequency of, organic meat $(\mathrm{OM})$ & & & & \begin{tabular}{|lr} 
social norms, & environmental \\
concern, green & behaviour, \\
perceived & consumer \\
effectiveness &
\end{tabular} \\
\hline $\begin{array}{lll}\text { Wachenheim } & \text { C.J. } & \text { and } \\
\text { VanWechel T. } & & \end{array}$ & 2004 & $\begin{array}{l}\text { Assesses whether WTP for food products can be } \\
\text { motivated by (label) information about } \\
\text { environmental effects of the use of GM } \\
\text { ingredients }\end{array}$ & 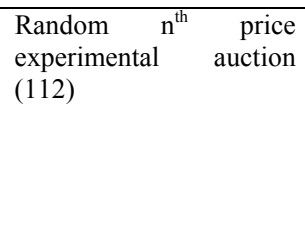 & Sealed bid & & $\begin{array}{l}\text { WTP on two variations of each } \\
\text { of the three products, one with a } \\
\text { standard NutriFacts label and } \\
\text { one with an additional label } \\
\text { indicating "This product does not } \\
\text { contain genetically modified } \\
\text { ingredients" }\end{array}$ \\
\hline Wang Q. and Sun J. & 2003 & $\begin{array}{l}\text { Assesses the market potential for organic apples } \\
\text { and milk, examining consumer evaluation of } \\
\text { major product attributes and their trade-offs }\end{array}$ & $\begin{array}{l}\text { Mail survey }(1659,519 \\
\text { returned) }\end{array}$ & $\begin{array}{l}\text { Conjoint analysis } \\
\text { Ordinary Least Squares }\end{array}$ & $\begin{array}{l}\text { Product variants (milk } \\
\text { and apples) }\end{array}$ & $\begin{array}{l}\text { Product attributes: production } \\
\text { method, location, certification, } \\
\text { price, container size }\end{array}$ \\
\hline
\end{tabular}


TABLE B.1.5: Empirical studies: Perception of labels

\begin{tabular}{|c|c|c|c|c|c|c|}
\hline Authors & Year & Purpose of the study & Survey method & Methodology & Dependent variable(s) & Explanatory variables \\
\hline Armah P.W. & 2002 & $\begin{array}{l}\text { Determines the variables that } \\
\text { influence eco-label use in organic } \\
\text { produce markets }\end{array}$ & $\begin{array}{l}\text { Consumer intercept direct } \\
\text { interviews (512, } 212 \text { usable) }\end{array}$ & Logit estimates & $\begin{array}{l}\text { Bivariate variable: Yes/No } \\
\text { questions about whether or } \\
\text { not respondents (producers, } \\
\text { consumers) rely on labels }\end{array}$ & $\begin{array}{l}\text { Socio-economic characteristics } \\
\text { Organic consumption behaviour }\end{array}$ \\
\hline Blend J. and van Ravensway E. & 1998 & $\begin{array}{l}\text { Examines potential consumer } \\
\text { demand for ecolabelled apples and } \\
\text { how different types of ecolabel } \\
\text { might affect consumer demand }\end{array}$ & $\begin{array}{l}\text { Phone interviews } \\
\text { interviews completed })\end{array}$ & $\begin{array}{llr}\text { Simulated } & \text { market: } & \text { two } \\
\text { scenarios } & \text { presented } & \text { to } \\
\text { respondents } & & \end{array}$ & $\begin{array}{l}\text { No model estimated } \\
\text { Choice between IPM and } \\
\text { ECO labels }\end{array}$ & $\begin{array}{l}\text { Demographics (age, income, } \\
\text { education), motivations, buy } \\
\text { organic yes/no }\end{array}$ \\
\hline Caswell J.A. & 2000 & $\begin{array}{l}\text { Discusses the reasons for the } \\
\text { adoption of labelling policies }\end{array}$ & & & & \\
\hline Caswell J.A. and Mojduszka E.M. & 1996 & $\begin{array}{l}\text { Discusses the economic rationales } \\
\text { for labelling policies and the issues } \\
\text { related to how the success or } \\
\text { failure of these policies should be } \\
\text { judged }\end{array}$ & & & & \\
\hline Conner D. and Christy R. & 2004 & $\begin{array}{l}\text { Reports the results of a survey and } \\
\text { experimental auction on } \\
\text { consumers preferences for } \\
\text { organic standards }\end{array}$ & Direct interviews (123) & $\begin{array}{l}\text { Contingent Valuation (open- } \\
\text { ended WTP elicitation) } \\
\text { English auction }\end{array}$ & & $\begin{array}{l}\text { Socio-economic } \\
\text { Attitudes and shopping habits } \\
\text { WTP } \\
\begin{array}{l}\text { Understanding of organic } \\
\text { (auction) }\end{array}\end{array}$ \\
\hline Grankvist G., Dahlstrand U.and Biel A. & 2004 & $\begin{array}{l}\text { Assesses the effect of two } \\
\text { labelling schemes on consumers' } \\
\text { preferences }\end{array}$ & $\begin{array}{l}\text { Computer-based experiment } \\
\text { (40) }\end{array}$ & $\begin{array}{l}\text { Laboratory } \\
\text { choice between pairs of } \\
\text { products }\end{array}$ & & Environmental concern \\
\hline Grolleau G. and Caswell J.A. & 2005 & $\begin{array}{l}\text { Presents a model where the level } \\
\text { of search and experience attributes } \\
\text { influence the likelihood of } \\
\text { production of eco-friendly } \\
\text { products }\end{array}$ & Theoretical paper & $\begin{array}{l}\text { Theoretic model of } \\
\text { interaction between product } \\
\text { attributes in determining the } \\
\text { likelihood of eco-friendly } \\
\text { production }\end{array}$ & & \\
\hline Jahn G., Schramm M.and Spiller A. & 2005 & $\begin{array}{l}\text { Analyses the core structure of } \\
\text { certification systems and tries to } \\
\text { evaluate the respective instruments }\end{array}$ & Model of audit effectiveness & & & \\
\hline
\end{tabular}




\begin{tabular}{|c|c|c|c|c|c|c|}
\hline & & to enhance audit quality & & & & \\
\hline $\begin{array}{l}\text { Johnston R.J., Wessells C.R., Donath H. } \\
\text { and Asche F. }\end{array}$ & 2001 & $\begin{array}{lcc}\text { Investigates } & \text { differences } & \text { in } \\
\text { consumer } & \begin{array}{c}\text { preferences } \\
\text { for }\end{array} \\
\text { ecolabelled } & \text { seafood across } & \text { the } \\
\text { United States and Norway } & \end{array}$ & $\begin{array}{l}\text { Contingent choice telephone } \\
\text { survey (1640 in the US; } \\
2039 \text { in Norway) }\end{array}$ & Choice model & Product alternatives & 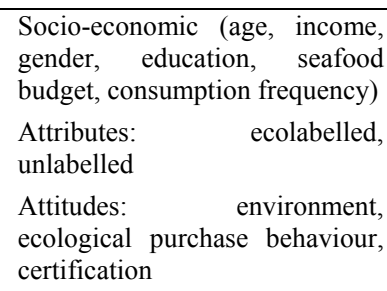 \\
\hline Roosen J., Lusk J.L. and Fox J.A. & 2001 & $\begin{array}{l}\text { Analyses consumer preferences for } \\
\text { alternative beef labelling strategies }\end{array}$ & $\begin{array}{l}\text { Mail surveys in France, } \\
\text { Germany, UK (1000 in each } \\
\text { country, } 76,43 \text {,and105 } \\
\text { usable respectively) }\end{array}$ & $\begin{array}{ll}\begin{array}{l}\text { Double bounded } \\
\text { models }\end{array} & \\
\text { Ordered probit model } & \end{array}$ & $\begin{array}{l}\text { Demand for mandatory } \\
\text { labelling } \\
\text { Importance of brand } \\
\text { Importance of origin }\end{array}$ & 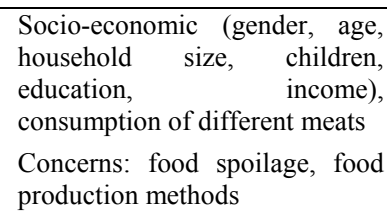 \\
\hline van Ravenswaay E.O. and Blend J.R. & 1997 & $\begin{array}{l}\text { Examines the potential for } \\
\text { ecolabelling as a mean to enhance } \\
\text { revenues from the adoption of } \\
\text { environmental technologies in } \\
\text { agriculture: ecolabelling } \\
\text { programmes and legal constraints, } \\
\text { necessary conditions for an } \\
\text { ecolabelling programme to } \\
\text { generate revenues sufficient to } \\
\text { encourag technology adoption, } \\
\text { consumer acceptance for } \\
\text { ecolabelled products }\end{array}$ & & & & \\
\hline
\end{tabular}


TABLE B.2.1: Main results: Real market data/experimental economics

\begin{tabular}{|c|c|c|c|c|}
\hline Authors & Year & Main results & Environmental and health factors & Other factors \\
\hline $\begin{array}{l}\text { Galloway K., Bailey D.V., "A Rose by Another } \\
\text { Name: An Objective Analysis of an Established } \\
\text { Market for Credence Attributes" }\end{array}$ & 2005 & $\begin{array}{l}\text { Positive average WTP bids for the cause coffee, } \\
\text { lowest for organic, greater for fair trade and shade- } \\
\text { grown. }\end{array}$ & $\begin{array}{l}\text { Effects on WTP for cause coffee } \\
\text { donate to environmental causes (-) }\end{array}$ & $\begin{array}{l}\text { Effects on WTP for cause coffee } \\
\text { Age }(+) \text { Married }(-) \\
\text { Health consciousness }(+) \text {, life satisfaction }(+) \text {, } \\
\text { life happiness }(-)\end{array}$ \\
\hline $\begin{array}{l}\text { Gifford K., Bernard J.C., Toensmeyer U.C., Bacon } \\
\text { R., "An Experimental Investigation of Willingness } \\
\text { to Pay for Non-GM and Organic Food Products" }\end{array}$ & 2005 & $\begin{array}{l}\text { Substantial untapped markets exist for GM and } \\
\text { organic products, with over half of respondents } \\
\text { bidding higher for one or both. }\end{array}$ & $\begin{array}{l}\text { Opinion on organic: 1-neg, 5-pos }(+) \\
\text { Opinion on GM (same range): }(-)\end{array}$ & $\begin{array}{l}\text { Organic: Age }(+) \text {, Income (-), education }(+) \\
\text { Non-GM: education (-), read label 1-never, 5- } \\
\text { always (-) }\end{array}$ \\
\hline $\begin{array}{l}\text { Glaser L.K., Thompson G.D., "Demand for } \\
\text { Organic and Conventional Frozen Vegetables" }\end{array}$ & 1998 & $\begin{array}{l}\text { Elasticities estimates suggest that consumers were } \\
\text { quite sensitive to own price changes in organic frozen } \\
\text { vegetables, 2-3 times more than conventional } \\
\text { counterparts. Some asymmetry in substitution } \\
\text { between organic and conventional is evident }\end{array}$ & & $\begin{array}{l}\text { Effects on consumption } \\
\text { Organic own price elasticities: from }-1.63 \text { to } \\
2.268 \\
\text { Expenditure elasticities: from } 0.778 \text { to } 1.489\end{array}$ \\
\hline $\begin{array}{l}\text { Glaser L.K., Thompson G.D., "Demand for } \\
\text { Organic and Conventional Beverage Milk" }\end{array}$ & 2000 & $\begin{array}{l}\text { Price premiums for organic milk averaged } 60 \% \text { of } \\
\text { branded prices and } 75 \% \text { of private label prices. Own } \\
\text { price elasticities suggest considerable response to } \\
\text { lower organic prices, although decreasing with } \\
\text { expenditure shares. Cross price elasticities indicate } \\
\text { substitution between organic and conventional but } \\
\text { with asymmetry in response }\end{array}$ & & $\begin{array}{l}\text { Effects on consumption } \\
\text { Organic own price elasticities: from }-3.637 \text { to - } \\
9.733 \\
\text { Cross-price elasticities: organic and branded (+), } \\
\text { organic and private label mostly (-) } \\
\text { Expenditure elasticities: from }-8.678 \text { to }-2.807\end{array}$ \\
\hline $\begin{array}{l}\mathrm{Hu} \text { Y., Mc Cluskey J.J., Durham C.A., } \\
\text { "Consumers' Willingness to Pay for Washington } \\
\text { Apples with Respect to Sensory Attributes" }\end{array}$ & 2005 & $\begin{array}{l}\text { Firmer and sweeter apples induce more WTP. Age is } \\
\text { an important factor affecting WTP for apples. } \\
\text { Education, eating frequency, and race affect WTP in } \\
\text { the Gala model but not the Red Delicious. Other } \\
\text { variables such as gender, annual household income } \\
\text { level, and whether they buy organic food, are not } \\
\text { significant }\end{array}$ & & $\begin{array}{l}\text { Effects on WTP } \\
\text { Firmness }(+) \text {, sweetness }(+) \text {, age }(-) \text {, education } \\
(+) \text { consumption frequency }(+)\end{array}$ \\
\hline $\begin{array}{l}\text { Lusk J.L., Daniel M.S., MarkD.R., Lusk C.L., } \\
\text { "Alternative Calibration and Auction Institutions } \\
\text { for Predicting Consumer Willingness to Pay for } \\
\text { Nongenetically Modified Corn Chips" }\end{array}$ & 2001 & $\begin{array}{l}\text { In a small and unrepresentative sample, } 70 \% \text { of } \\
\text { student participants were unwilling to pay to } \\
\text { exchange a bag of chips made from genetically } \\
\text { modified ingredients for a bag of chips made from } \\
\text { non-genetically modified ingredients. However, } 20 \% \\
\text { of respondents were willing to pay at least } \$ 0.25 / \mathrm{oz} \text {. } \\
\text { for the exchange }\end{array}$ & $\begin{array}{l}\text { Effects on WTP for non-GM chips } \\
\text { Exercise on regular basis }(1=\text { yes, } 0=\text { no })(+) \\
\text { GM concern }(+)\end{array}$ & $\begin{array}{l}\text { Effects on WTP for non-GM chips } \\
\text { Consumption frequency (-) }\end{array}$ \\
\hline $\begin{array}{l}\text { Nayga R.M., Tepper B.J., Rosenzweig L., } \\
\text { "Assessing the importance of health and nutrition }\end{array}$ & 1999 & $\begin{array}{l}\text { Results of the models show that diet disease, } \\
\text { individual's race, region of residence, urbanisation, }\end{array}$ & $\begin{array}{l}\text { Effects on average daily intake } \\
\text { Own perceived healthier ( }+ \text { on dairy and poultry }\end{array}$ & $\begin{array}{l}\text { Effects on average daily intake } \\
\text { Male }(+)\end{array}$ \\
\hline
\end{tabular}




\begin{tabular}{|c|c|c|c|c|}
\hline $\begin{array}{l}\text { related factors on food demand: a variable } \\
\text { preference investigation" }\end{array}$ & & $\begin{array}{l}\text { education, and perceived importance of taste } \\
\text { influence the consumption of various food groups }\end{array}$ & $\begin{array}{l}\text { products) } \\
\text { Overweight ( }+ \text { on sugar) } \\
\text { Diet disease knowledge ( }+ \text { on fruit and vegetables) }\end{array}$ & $\begin{array}{l}\text { Non-metropolitan area (-) } \\
\text { Importance of taste (+ on dairies, meat, } \\
\text { sugar/sweets) }\end{array}$ \\
\hline $\begin{array}{l}\text { Roosen J., Fox J.A., Hennessy D.A., Schreiber A., } \\
\text { "Consumers' Valuation of Insecticide Use } \\
\text { Restrictions: An Application to Apples" }\end{array}$ & 1998 & $\begin{array}{l}\text { Consumer perceptions of product attributes } \\
\text { change if pesticides are removed from production, and } \\
\text { this is reflected in WTP changes. WTP is shown to be } \\
\text { income-elastic }\end{array}$ & $\begin{array}{l}\text { Effects on WTP for reduced pesticide use } \\
\text { No pesticide }(+) \\
\text { Pesticide concern (+ on preference for reduced } \\
\text { pesticide use) }\end{array}$ & $\begin{array}{l}\text { Effects on WTP for reduced pesticide use } \\
\text { Income }(+) \text {, children }(+) \text {, consumption }(-) \text {, price } \\
(-)\end{array}$ \\
\hline $\begin{array}{l}\text { Thompson G.D., Glaser L.K., "National Demand } \\
\text { for Organic and Conventional Baby Food" }\end{array}$ & 2001 & $\begin{array}{l}\text { Results show high own price elasticities for organic } \\
\text { baby foods and low for conventional. These two } \\
\text { categories are substitutes }\end{array}$ & & $\begin{array}{l}\text { Effects on consumption } \\
\text { Own price elasticities: } \\
\text { organic: }-2.489 /-3.110 \text {; conventional: }-0.154 /- \\
0.165 \\
\text { Cross-price elasticities: } \\
\text { Organic/conventional: } 1.913\end{array}$ \\
\hline $\begin{array}{l}\text { Thompson G.D., Kidwell J., "Explaining the } \\
\text { Choice of Organic Produce: Cosmetic Defects, } \\
\text { Prices, and Consumer Preferences" }\end{array}$ & 1998 & $\begin{array}{l}\text { Shoppers at the specialty grocer were sensitive to } \\
\text { price differences between organic and conventional } \\
\text { items. Cosmetic defects had a small but significant } \\
\text { impact on the likelihood to buy organic }\end{array}$ & & $\begin{array}{l}\text { Effects on probability of purchasing organic } \\
\text { Age under } 18(+) \\
\text { Defects }(-) \text {, store }(1=\text { specialty, } 0=\text { coop) }(-) \\
\text { Effects on probability of shopping at specialty } \\
\text { store } \\
\text { Income }(+) \text {, buy certified organic }(-)\end{array}$ \\
\hline $\begin{array}{l}\text { Wier M., Morch Andersen L., Millock K., } \\
\text { O'Doherty Jensen K., Rosenqvist L., "Perceptions, } \\
\text { values and behaviour: The case of organic foods" }\end{array}$ & 2005 & $\begin{array}{l}\text { Household propensity to purchase organic foods } \\
\text { increases significantly with the declared importance } \\
\text { accorded to 'private good' attributes (i.e. taste). The } \\
\text { weight that households assign to public good } \\
\text { attributes (i.e. environment preservation) does not } \\
\text { contribute significantly to the explanation of } \\
\text { household organic budget share }\end{array}$ & $\begin{array}{l}\text { Effects on organic budget share } \\
\text { Pesticide residue health concern }(+)\end{array}$ & $\begin{array}{l}\text { Effects on organic budget share } \\
\text { Stated private good values (+); purchasing } \\
\text { barriers (trust in control, knowledge, interest in } \\
\text { organic) }(-) \text {; age }(+) \text {, } \\
\text { children }<14(+) \text {; teens }(15-18)(-) \text {; education }(+)\end{array}$ \\
\hline $\begin{array}{l}\text { York R., Hill Gossard M., "Cross-national meat } \\
\text { and fish consumption: exploring the effects of } \\
\text { modernization and ecological context" }\end{array}$ & 2004 & $\begin{array}{l}\text { Ecological conditions in a nation, such as resource } \\
\text { availability and climate, influence meat and fish } \\
\text { consumption. Additionally, indicators of } \\
\text { modernisation, particularly economic development, } \\
\text { influence the consumption of both meat and fish. } \\
\text { However, the effect of economic development on } \\
\text { consumption patterns is distinctly different among } \\
\text { geographic regions }\end{array}$ & $\begin{array}{l}\text { Effects on per-capita consumption } \\
\text { Land p.c. (+ for meat) } \\
\text { Water p.c. (+ for fish) }\end{array}$ & $\begin{array}{l}\text { Effects on per-capita consumption } \\
\text { GDP }(+)\end{array}$ \\
\hline
\end{tabular}




\section{TABLE B.2.2: Main Results: Hypothetical markets (e.g. willingness to pay)}

\begin{tabular}{|c|c|c|c|c|}
\hline Authors & Year & Main results & $\begin{array}{l}\text { Environmental and health } \\
\text { factors }\end{array}$ & Other factors \\
\hline $\begin{array}{l}\text { Ara S., "Consumer Willingness to } \\
\text { Pay for Multiple Attributes of } \\
\text { Organic Rice : A Case Study in the } \\
\text { Philippines" }\end{array}$ & 2003 & $\begin{array}{l}\text { Health risk is the primary concern for the choice of organic, while organic certification } \\
\text { is second. }\end{array}$ & $\begin{array}{l}\text { Effects on "choice of organic" } \\
\text { Reduced health risk }(+) \\
\text { Environmental quality }(+) \\
\text { Certification }(+)\end{array}$ & $\begin{array}{l}\text { Effects on "choice of organic" } \\
\text { Price (-), eating quality }(+) \text {, fair trade }(+)\end{array}$ \\
\hline $\begin{array}{l}\text { Baker G., "Consumer Preferences } \\
\text { for Food Safety Attributes in Fresh } \\
\text { Apples: Market Segments, } \\
\text { Consumer Characteristics, and } \\
\text { Marketing Opportunities" }\end{array}$ & 1999 & $\begin{array}{l}\text { Consumers expressed a broad preference for reduced pesticide usage. Four market } \\
\text { segments were identified corresponding to consumers: (a) who had a strong preference } \\
\text { for food safety, (b) who exhibited a more balanced desire for all product } \\
\text { characteristics, (c) who were extremely price-sensitive, and (d) who had a strong } \\
\text { preference for product quality }\end{array}$ & $\begin{array}{l}\text { Effects on utility (discrete choice) } \\
\text { Pesticide policy: conventional }(-), \\
\text { reduced pesticide }(+) \text {, organic }(+) \\
\begin{array}{l}\text { Certification: monitoring } \\
\text { certification }(+)\end{array}\end{array}$ & $\begin{array}{l}\text { Effects on utility (discrete choice) } \\
\text { Price (-) } \\
\text { Damage (-) }\end{array}$ \\
\hline $\begin{array}{l}\text { Batte M.T. et al., "Customer } \\
\text { Willingness to Pay for Multi- } \\
\text { Ingredient, Processed Organic Food } \\
\text { Products" }\end{array}$ & 2004 & $\begin{array}{l}\text { Nutrition and a desire to avoid pesticides were the primary motives for purchasing } \\
\text { organic foods, while high price and a perceived lack of variety were the most } \\
\text { important reasons for not purchasing. Estimates of willingness to pay for organic foods } \\
\text { suggest that consumers are willing to pay premium prices for organic foods, even those } \\
\text { with less than } 100 \% \text { organic ingredients }\end{array}$ & $\begin{array}{l}\text { Effects on WTP for organic } \\
\text { Health index (-) } \\
\text { Effects on discrete choice of cereals } \\
\text { Health index (+ for } 70 \% \text { organic) } \\
\text { Safety index }(+)\end{array}$ & $\begin{array}{l}\text { Effects on WTP for organic } \\
\text { Age }(+) \text {, income }(+) \text {, non-white }(+) \text {, female } \\
(+) \text {, specialty grocery shoppers }(+) \text {, children } \\
(+) \\
\text { Effects on discrete choice of organic cereals } \\
\text { Price }(-) \text {, children }(- \text { for } 70 \% \text { and } 100 \% \\
\text { organic), specialty grocery }(+)\end{array}$ \\
\hline $\begin{array}{l}\text { Boccaletti S., Nardella M., } \\
\text { "Consumer Willingness to Pay for } \\
\text { Pesticide-Free Fresh Fruit and } \\
\text { Vegetables in Italy" }\end{array}$ & 2000 & $\begin{array}{l}\text { Italian consumers were generally concerned about health risks from pesticides, with } \\
\text { only } 11 \% \text { of the respondents not willing to pay higher prices for pesticide-free fresh } \\
\text { fruits and vegetables. } \\
\text { The most relevant increase in the probability of a positive WTP was given by income } \\
\text { and individual perception of risk concern about pesticides, while higher education } \\
\text { increased the probability of a zero WTP }\end{array}$ & $\begin{array}{l}\text { Effects on WTP for pesticide-free } \\
\text { Pesticide concern index }(+)\end{array}$ & $\begin{array}{l}\text { Effects on WTP for pesticide free } \\
\text { Female }(+) \text {, education }(-), \text { income }(+)\end{array}$ \\
\hline $\begin{array}{l}\text { Buzby J.C., Ready R.C., Skees J.R., } \\
\text { "Contingent Valuation in Analysis: } \\
\text { A Case Study Residue Risk } \\
\text { Reduction Food Policy of a } \\
\text { Pesticide-Residue Risk Reduction" }\end{array}$ & 1995 & $\begin{array}{l}\text { Estimated benefits of banning a specific post-harvest pesticide used in fresh grapefruit } \\
\text { packinghouses outweigh costs: growers would be negatively affected, while } \\
\text { consumers' benefit from reduced risk would outweigh the increase in price.l }\end{array}$ & $\begin{array}{l}\text { Effects on WTP for pesticide-free } \\
\text { Pesticide concern }(+) \\
\text { Ban pesticides }(+)\end{array}$ & $\begin{array}{l}\text { Effects on WTP for pesticide-free } \\
\text { Income (-), age (-), }\end{array}$ \\
\hline $\begin{array}{l}\text { Byrne P.J., Toensmeyer } \\
\text { German } \text { C.C., } \\
\text { "Analysis of Consumer Attitude } \\
\text { Toward Organic Produce and }\end{array}$ & 1991 & $\begin{array}{l}\text { Higher education and males demonstrate negative effects on the organic alternative. } \\
\text { While higher-income households do not necessarily favour organic produce purchases, } \\
\text { their ability to pay for the higher-priced good is evident from their positive purchase } \\
\text { likelihood result. }\end{array}$ & & $\begin{array}{l}\text { Effects on "purchase organic"at higher } \\
\text { prices } \\
\text { Female (+), education }(-) \text {, income }(+)\end{array}$ \\
\hline
\end{tabular}




\begin{tabular}{|c|c|c|c|c|}
\hline Purchase Likelihood" & & & & \\
\hline $\begin{array}{l}\text { Cicia G., Del Giudice T., Scarpa R., } \\
\text { "Consumers' perception of quality } \\
\text { in organic food. A random utility } \\
\text { model under preference } \\
\text { heterogeneity and choice correlation } \\
\text { from rank-orderings" }\end{array}$ & 2002 & $\begin{array}{l}\text { Consumers who regularly buy organic food belong to a homogeneous segment. } \\
\text { Consumers perceive price as a quality signal and prefer the product certified by } \\
\text { reliable certification agencies }\end{array}$ & $\begin{array}{l}\text { Effects on discrete choice of organic } \\
\text { olive oil } \\
\text { Certification }(+)\end{array}$ & $\begin{array}{l}\text { Effects on discrete choice of organic olive oil } \\
\text { Price }(+) \text {, product origin }(-)\end{array}$ \\
\hline $\begin{array}{l}\text { Cranfield J.A.L., Magnusson E., } \\
\text { "Canadian Consumer's Willingness- } \\
\text { To-Pay for Pesticide Free Food } \\
\text { Products: An Ordered Probit } \\
\text { Analysis" }\end{array}$ & 2003 & $\begin{array}{l}\text { Over } 65 \% \text { of respondents would be willing to pay a } 1 \% \text { to } 10 \% \text { premium relative to a } \\
\text { conventional food product. Five per cent of respondents would be willing to pay more } \\
\text { than a } 20 \% \text { premium. Health and environmental concerns, willingness to switch } \\
\text { grocery stores and youth are important characteristics of consumers who would be } \\
\text { willing to pay higher premiums. Distribution channels geared towards health food } \\
\text { stores (or health food centres within grocery stores) are likely targets for Pesticide Free } \\
\text { Production (PFP) certified food products }\end{array}$ & $\begin{array}{l}\text { Effects on WTP for pesticide-free } \\
\text { production }(P F P) \\
\text { Pesticide concern }(+)\end{array}$ & $\begin{array}{l}\text { Effects on WTP for pesticide-free production } \\
(P F P) \\
\text { Try new products }(-) \text {, switch store to buy PFP } \\
(+) \\
\text { Age (less than } 36,+)\end{array}$ \\
\hline $\begin{array}{l}\text { Durham C.A., Andrade D., "Health } \\
\text { vs. Environmental Motivation in } \\
\text { Organic Preferences and Purchases" }\end{array}$ & 2005 & $\begin{array}{l}\text { Both health and the environment are motivations for organic preferences and } \\
\text { purchasing, but environmental motivations are more influential in determining higher } \\
\text { levels of purchases }\end{array}$ & $\begin{array}{l}\text { Effects on preference for organic } \\
\text { fresh fruit and vegetables } \\
\text { EEPB }(+) \\
\text { Health environmental sensitivity }(+)\end{array}$ & $\begin{array}{l}\text { Effects on preference for organic fresh fruit } \\
\text { and vegetables } \\
\text { Age (-), price importance (-) }\end{array}$ \\
\hline $\begin{array}{l}\text { Eom Y.S., "Pesticide Residue Risk } \\
\text { and Food Safety Valuation: A } \\
\text { Random Utility Approach" }\end{array}$ & 1994 & $\begin{array}{l}\text { Results from a pilot survey suggest a clear linkage between perception and behaviour } \\
\text { in response to new risk information. Consumers' stated preferences for safer produce } \\
\text { were influenced by price differences and perceived risks }\end{array}$ & $\begin{array}{l}\text { Effects on purchase intention for } \\
\text { safer produce } \\
\text { Risk reduction from safer prod. }(+) \\
\text { Pesticide health risk concern }(+) \\
\text { Pesticide negative effect on env }(+)\end{array}$ & $\begin{array}{l}\text { Effects on purchase intention for safer } \\
\text { produce } \\
\text { Price increase (-), education (-) } \\
\text { regularly purchase organic }(+)\end{array}$ \\
\hline $\begin{array}{l}\text { Gil J. M, Gracia A., Sanchez M., } \\
\text { "Market segmentation and } \\
\text { willingness to pay for organic } \\
\text { products in Spain" }\end{array}$ & 2000 & $\begin{array}{l}\text { Consumers concerned about healthy diet and environmental degradation are the most } \\
\text { likely to buy organic food, and are willing to pay a high premium. Organic attributes } \\
\text { are easily identified in perishable products as the premium consumers would pay for } \\
\text { organic meat, fruits, and vegetables is higher. }\end{array}$ & & \\
\hline $\begin{array}{l}\text { Govindasamy R., Italia J., } \\
\text { "Consumer Response to Integrated } \\
\text { Pest Management and Organic } \\
\text { Agriculture: An Econometric } \\
\text { Analysis" }\end{array}$ & 1997 & $\begin{array}{l}\text { Income was found to be the most significant determinant of willingness-to-purchase } \\
\text { IPM grown produce. Participants with higher annual incomes were more likely to } \\
\text { express an interest in purchasing IPM produce and also appeared less likely to strictly } \\
\text { purchase conventional produce. Those who frequently purchase organic produce, those } \\
\text { who visit farmers markets and those who live in suburban areas were all found to be } \\
\text { more likely to purchase IPM grown produce. The results also indicate that women, } \\
\text { those with higher annual incomes, younger individuals, and those who frequently } \\
\text { purchase organic produce are all more likely to pay a premium for both IPM and } \\
\text { organically grown produce }\end{array}$ & & $\begin{array}{l}\text { Effects on willingness to purchase IPM } \\
\text { Age }>65 \quad(-) \text {, income }(+) \text {, shop many } \\
\text { supermarkets }(-) \\
\text { urban }(-) \text {, rural }(-) \text {, primary grocery shopper } \\
(+) \\
\text { Effects on WTP }>10 \% \text { for organic } \\
\text { Female }(+) \text {, age }(+) \text {, income }(+) \text {, education }(- \\
) \text {, regularly purchase organic }(+) \text {, household } \\
\text { size }(-) \text {, try new products }(+) \text {, heard of IPM } \\
(+)\end{array}$ \\
\hline
\end{tabular}




\begin{tabular}{|c|c|c|c|c|}
\hline $\begin{array}{lcr}\text { Govindasamy } & \text { R., Italia } & \text { J., } \\
\text { "Predicting } & \text { Consumer } & \text { Risk } \\
\text { Perceptions } & \text { Towards } & \text { Pesticide } \\
\text { Residue: a Logistic Analysis" }\end{array}$ & 1998 & $\begin{array}{l}\text { Women, households with several children, and individuals over } 35 \text { years old are more } \\
\text { likely to have high levels of risk aversion. Households with higher levels of income } \\
\text { and education exhibited lower risk aversions towards pesticide residues }\end{array}$ & & $\begin{array}{l}\text { Effects on "highly adverse to pesticides" } \\
\text { Female }(+), \text { Age }<35(-) \text {, education }(-), \\
\text { children }(+) \text { suburban }(+), \text { income }(-)\end{array}$ \\
\hline $\begin{array}{l}\text { Govindasamy R., Italia J., } \\
\text { "Predicting Willingness-to-Pay a } \\
\text { Premium for Organically Grown } \\
\text { Fresh Produce" }\end{array}$ & 1999 & $\begin{array}{l}\text { Results indicate that women, those with higher annual incomes, younger individuals, } \\
\text { and those who usually or always purchase organic produce are all more likely to pay a } \\
\text { premium for organic produce. The results also indicate that the likelihood of paying a } \\
\text { premium for organic produce decreases with the number of individuals living in the } \\
\text { household }\end{array}$ & $\begin{array}{l}\text { Effects on WTP }>10 \% \\
\text { Use food safety media reports }(+)\end{array}$ & $\begin{array}{l}\text { Effects on WTP }>10 \% \\
\text { Female }(+) \text {, income }(+) \text {, age }(-) \text {, education }(-) \\
\text { Regularly purchase organic }(+) \text {, household } \\
\text { size }(-) \text {, heard of IPM }(+) \text {, try new products } \\
(+),\end{array}$ \\
\hline $\begin{array}{l}\text { Govindasamy R., Italia J., Adelaja } \\
\text { A., "Predicting Willingness-to-Pay a } \\
\text { Premium for Integrated Pest } \\
\text { Management Produce: A Logistic } \\
\text { Approach" }\end{array}$ & 2001 & $\begin{array}{l}\text { Results indicate that women, those with higher annual incomes, younger individuals, } \\
\text { and those who frequently purchase organic produce are all more likely to pay a } \\
\text { premium for 1PM produce }\end{array}$ & $\begin{array}{l}\text { Effects on WTP }>10 \% \\
\text { Pesticides risk aversion }(+)\end{array}$ & $\begin{array}{l}\text { Effects on WTP }>10 \% \\
\text { Female }(+) \text {, age }(+) \text {, income }(+) \text {, household } \\
\text { size }(-), \text { regularly purchase organic }(+)\end{array}$ \\
\hline $\begin{array}{l}\text { Groff A.J., } \quad \text { Kreider } \quad \text { C.R., } \\
\text { Toensmeyer U.C., "Analysis of the } \\
\text { Delaware Market } \\
\text { Grown Produce" Organically }\end{array}$ & 1993 & $\begin{array}{l}\text { Younger respondents, female respondents, and those with lower education levels were } \\
\text { more likely to feel organics are a better produce than conventionally grown produce }\end{array}$ & & $\begin{array}{l}\text { Effects on consumer's better rating of } \\
\text { organic vs conventional } \\
\text { Age (-), education }(-), \text { female }(+)\end{array}$ \\
\hline $\begin{array}{l}\text { Hearne R.R., Volcan M.M., "The } \\
\text { Use of Choice Experiments to } \\
\text { Analyze Consumer Preferences for } \\
\text { Organic Produce in Costa Rica" }\end{array}$ & 2002 & $\begin{array}{l}\text { The study indicates a positive willingness to pay among Costa Rican consumers for } \\
\text { certified food safety and organic production practices }\end{array}$ & & $\begin{array}{l}\text { Effects on certified labelled (organic) } \\
\text { product } \\
\text { Size }(+) \text {, appearance }(+) \text {, price (-), education } \\
(+)\end{array}$ \\
\hline $\begin{array}{l}\text { Huang C.L., "Consumer preferences } \\
\text { and attitudes towards organically } \\
\text { grown produce" }\end{array}$ & 1996 & $\begin{array}{l}\text { Consumers who are nutritionally conscious, concerned about the use of pesticides, and } \\
\text { desire residues-free produce have a higher propensity to prefer organically grown } \\
\text { produce }\end{array}$ & $\begin{array}{l}\text { Effects on preference for organic } \\
\text { produce } \\
\text { Pesticide use concern }(+) \\
\text { Ban pesticides }(+) \text {, Test and certif. } \\
(+) \\
\text { Nutr. value important }(+)\end{array}$ & $\begin{array}{l}\text { Effects on preference for organic produce } \\
\text { Low price important (-) } \\
\text { Effects on willingness to accept sensory } \\
\text { defect } \\
\text { Appearance important (-), low price (+), } \\
\text { education (+), household size (+), income (-) }\end{array}$ \\
\hline $\begin{array}{l}\text { Jolly D.A., "Determinants of } \\
\text { Organic Horticultural Products } \\
\text { Consumption Based on a Sample of } \\
\text { California Consumers" }\end{array}$ & 1991 & & $\begin{array}{l}\text { Effects on WTP for organic } \\
\text { Concern for artificial colouring }(+)\end{array}$ & $\begin{array}{l}\text { Effects on WTP for organic } \\
\text { Education }(+) \text {, married }(-) \text {, white collar }(+), \\
\text { income }(+) \text {, female }(+)\end{array}$ \\
\hline $\begin{array}{l}\text { Larue B., West G.E., Gendron C., } \\
\text { Lambert R., "Consumer Response } \\
\text { to Functional Foods Produced by } \\
\text { Conventional, Organic, or Genetic }\end{array}$ & 2004 & $\begin{array}{l}\text { Results indicate that many Canadian consumers will avoid GM foods, regardless of the } \\
\text { presence of functional health properties. For others, the introduction of GM functional } \\
\text { plant foods should increase acceptance of GM production methods, but many } \\
\text { consumers will likely avoid functional foods derived from GM animals. The organic }\end{array}$ & $\begin{array}{l}\text { Effects on utility (discrete choice } \\
\text { among conventional., GM, organic) } \\
\text { Health positive properties }(+)\end{array}$ & \\
\hline
\end{tabular}




\begin{tabular}{|c|c|c|c|c|}
\hline Manipulation" & & food industry could also profit from the introduction of organic functional foods & & \\
\hline $\begin{array}{l}\text { Loureiro M.L., } \text { Hine S., } \\
\text { "Discovering Niche Markets: A } \\
\text { Comparison of Consumer } \\
\text { Willingness to Pay for Local } \\
\text { (Colorado Grown), Organic, and } \\
\text { GMO-Free Products" }\end{array}$ & 2002 & $\begin{array}{l}\text { Willingness-to-pay estimates show a higher premium for the "Colorado grown" } \\
\text { attribute. This attribute affords the potato producer with the highest consumer } \\
\text { acceptance and premium (relative to organic and GMO-free) }\end{array}$ & & $\begin{array}{l}\text { Effects on WTP for organic potatoes } \\
\text { Age }(-) \text {, upper class }(+) \text {, freshness concern } \\
(+), \\
\text { nutrition concern }(+)\end{array}$ \\
\hline $\begin{array}{l}\text { Loureiro M.L., Lotade J., "Do fair } \\
\text { trade and eco-labels in coffee wake } \\
\text { up the consumer conscience?" }\end{array}$ & 2005 & $\begin{array}{l}\text { Consumers are very receptive towards both fair trade and shade grown coffee labels, } \\
\text { and consequently are willing to pay higher premiums for these labelling programmes } \\
\text { than for the organic coffee }\end{array}$ & $\begin{array}{l}\text { Effects on WTP for organic coffee } \\
\begin{array}{l}\text { Environmental } \\
\text { creation }(+)\end{array} \text { concern } \quad \text { vs. job }\end{array}$ & $\begin{array}{l}\text { Effects on WTP for organic coffee } \\
\text { Income (+), Age (-), importance of welfare of } \\
\text { future generations (-) }\end{array}$ \\
\hline $\begin{array}{l}\text { Loureiro M.L., McCluskey J.J., } \\
\text { Mittelhammer R.C., "Assessing } \\
\text { Consumer Preferences for Organic, } \\
\text { Eco-labeled, and Regular Apples" }\end{array}$ & 2001 & $\begin{array}{l}\text { Eco-labeled apples are less desirable than organic when food safety, the environment, } \\
\text { and children's needs are considered. Characteristics that may be expected to positively } \\
\text { affect the decision to buy eco-labelled apples relative to regular apples actually have } \\
\text { the opposite effect with the inclusion of the organic alternative. When considering all } \\
\text { three choices, the eco-labelled product is found to be an intermediate choice among } \\
\text { consumers }\end{array}$ & $\begin{array}{l}\text { Effects on utility (discrete choice for } \\
\text { organic) } \\
\begin{array}{l}\text { Environmental concern } \quad \text { vs. job } \\
\text { creation }(+)\end{array} \\
\text { Food safety vs. appearance }(+)\end{array}$ & $\begin{array}{l}\text { Effects on utility (discrete choice for organic) } \\
\text { Children }(+) \text {, income }(+) \text {, family size }(-) \\
\text { female }(+)\end{array}$ \\
\hline $\begin{array}{l}\text { Loureiro M.L., McCluskey J.J., } \\
\text { Mittelhammer R.C., "Willingness to } \\
\text { Pay for Sustainable Agriculture } \\
\text { Products" }\end{array}$ & 1999 & $\begin{array}{l}\text { When consumers choose from among organic, sustainable agriculture and } \\
\text { conventional apples, the sustainable agriculture choice is an intermediate choice when } \\
\text { food safety, the environment and children's needs are considered }\end{array}$ & 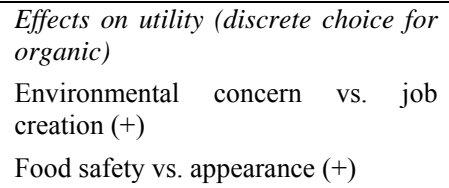 & $\begin{array}{l}\text { Effects on utility (discrete choice for organic) } \\
\text { Children }(+) \text {, family size }(-)\end{array}$ \\
\hline $\begin{array}{l}\text { Magnusson E., Cranfield A.L., } \\
\text { "Consumer Demand for Pesticide } \\
\text { Free Food Products in Canada: A } \\
\text { Probit Analysis" }\end{array}$ & 2005 & $\begin{array}{l}\text { Consumers show a strong interest in food products containing grains and oilseeds } \\
\text { produced with pesticide-free practices. Preference for these products increases with } \\
\text { environmental and pesticide concern }\end{array}$ & $\begin{array}{l}\text { Effects on utility (discrete choice for } \\
\text { PFPs) } \\
\text { Health concern }(+) \\
\text { Environmental concern }(+)\end{array}$ & $\begin{array}{l}\text { Effects on utility (discrete choice for PFPs) } \\
\text { Age }<36(+) \text {, switch store to buy PFPs }(+) \text {, } \\
\text { education }(-) \text {, income }(+) \text {, willing to pay } \\
\text { premium for PFPs }(+)\end{array}$ \\
\hline $\begin{array}{l}\text { Misra S., Huang C.L., Ott S.L., } \\
\text { "Consumer Willingness to Pay for } \\
\text { Pesticide-Free Fresh Produce" }\end{array}$ & 1991 & $\begin{array}{l}\text { The analysis suggests that the more the consumers were concerned about health effects } \\
\text { of pesticide residues on fresh produce, the more likely they would be willing to pay a } \\
\text { higher premium for certified-RFP produce. The results also show that consumers' } \\
\text { attitudes towards testing and certification and future expectations play a significant } \\
\text { role in influencing their willingness to pay }\end{array}$ & $\begin{array}{l}\text { Effects on WTP for certified pesticide } \\
\text { residues-free produce } \\
\text { Pesticides concern index }(+) \\
\text { Importance of testing and cert. }(+)\end{array}$ & $\begin{array}{l}\text { Effects on WTP for certified pesticide } \\
\text { residues-free produce } \\
\text { Age }(+) \text {, education }(-) \text {, income }(+) \text {, future } \\
\text { financial status }(+) \text {, white }(+)\end{array}$ \\
\hline $\begin{array}{l}\text { Roheim C.A., Johnston R.J., Greer } \\
\text { J., Donath H., "Consumer } \\
\text { Preferences for Ecolabeled Seafood: } \\
\text { Results of a Connecticut Survey" }\end{array}$ & 2005 & $\begin{array}{l}\text { Results suggest that consumers are unwilling to choose a less-favoured species (i.e. to } \\
\text { sacrifice taste) based solely on the presence of an ecolabel }\end{array}$ & & $\begin{array}{l}\text { Effects on utility } \\
\text { Price (-), eco-label }(+)\end{array}$ \\
\hline $\begin{array}{l}\text { Scarpa R., Spalatro F., Canavari M., } \\
\text { "Investigating preferences for } \\
\text { environment friendly production } \\
\text { practices: taste segments for organic }\end{array}$ & 2002 & $\begin{array}{l}\text { The distribution of taste intensity for organic, integrated crop management and quality } \\
\text { certification share a common correlation structure. The largest price differential is } \\
\text { commanded by organic potato production. Only } 23 \% \text { of the share of households in }\end{array}$ & & $\begin{array}{l}\text { Effects on utility } \\
\text { Age }(-) \text {, household size }(+), \text { large size }(+) \text {, } \\
\text { poor appearance }(-) \text {, price }(-)\end{array}$ \\
\hline
\end{tabular}




\begin{tabular}{|c|c|c|c|c|}
\hline $\begin{array}{l}\text { and integrated crop management in } \\
\text { Italian households" }\end{array}$ & & Italy is not interested in environmentally friendly production practices & & \\
\hline $\begin{array}{l}\text { Shuzzler A., Govindasamy R., } \\
\text { Adelaja A., "A Comparative } \\
\text { Evaluation of Organic Produce } \\
\text { Consumers in New Jersey to New } \\
\text { York and Pennsylvania" }\end{array}$ & 2003 & $\begin{array}{l}\text { Relevant differences between consumer's preferences for organic produce emerge in } \\
\text { the two states }\end{array}$ & & $\begin{array}{l}\text { Effects on "frequently buy organic" } \\
\text { Household size }(+) \text {, age }(+) \text {, income }(+) \\
\text { Perceived high quality of organic }(+) \\
\text { Perceived higher variety for organic }(-) \\
\text { Switch supermarkets to buy organic }(+) \\
\text { Check labels }(+), \text { heard of IPM }(+)\end{array}$ \\
\hline 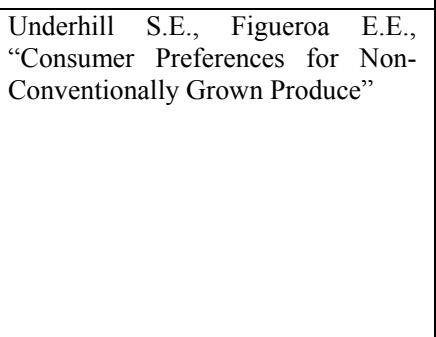 & 1996 & $\begin{array}{l}71 \% \text { of respondents stated they believed that pesticide residues in food present a } \\
\text { serious or moderate health hazard to consumers. In addition, } 74 \% \text { believed that } \\
\text { pesticides pose a serious or moderate hazard to the environment, and } 64 \% \text { felt there } \\
\text { was a serious or moderate hazard to farm workers. Results indicate there is a positive } \\
\text { information effect for likelihood of purchasing for all of the labels, and this effect is } \\
\text { statistically significant for all of the labels except for "Certified Pesticide Residue- } \\
\text { Free". The magnitude of the information effect for the Grown with IPM label was } \\
\text { considerably higher than for the other labels, suggesting that there might be substantial } \\
\text { pay-offs for informing consumers about this label }\end{array}$ & & $\begin{array}{l}\text { Effects on purchase of organic/certified } \\
\text { organic } \\
\text { Information }(+) \text {, rural/suburban (-), age (-), } \\
\text { female }(+) \text {, income }(+) \\
\text { Effects on WTP for organic/certified organic } \\
\text { Rural/suburban (-), age }(-), \text { income }(+)\end{array}$ \\
\hline $\begin{array}{llr}\text { Veeman M., } & \text { Adamowicz } & \text { W., } \\
\text { "Consumer's } & \text { Perceptions } & \text { of } \\
\text { Environmental } & \text { Risks and } & \text { the } \\
\text { Demand for Food Safety" } & \end{array}$ & 2000 & $\begin{array}{l}\text { Albertans were more concerned about pesticide use in food production than about the } \\
\text { use of hormones. In contingent valuation questions, more Albertans wish to restrict } \\
\text { pesticide use (relative to a base case of not restricting either hormones or pesticides). } \\
\text { They tended to persist in these choices in the face of potential increases in food costs, } \\
\text { reflecting a higher level of concern with pesticides than with hormones }\end{array}$ & $\begin{array}{l}\text { Effects on utility (choice of milk) } \\
\text { Use of recombinant Bovine } \\
\text { Somatotropine (BST) (-) }\end{array}$ & $\begin{array}{l}\text { Effects on utility (choice of milk) } \\
\text { Price }(-) \text {, freshness }(+) \text {, age }(+) \text {, female }(+), \\
\text { education }(+ \text { for skimmed), BST knowledge } \\
(+)\end{array}$ \\
\hline $\begin{array}{l}\text { Verhoef P.C., } \\
\text { purchases of oxplaining } \\
\text { Dutch consumers" }\end{array}$ & 2005 & $\begin{array}{l}\text { Consumers' purchase of organic meat is based on both 'rational' economic motives } \\
\text { and emotional motives }\end{array}$ & $\begin{array}{l}\text { Effects on choice/purchasing } \\
\text { frequency of organic meat } \\
\text { Green behaviour }(+) \\
\text { Consumer's effectiveness in } \\
\text { changing environment }(+/+)\end{array}$ & $\begin{array}{l}\text { Effects on choice/purchasing frequency of } \\
\text { organic meat } \\
\text { Quality perception }(+) \text {, price perception (-/-), } \\
\text { distribution assortment of OM }(+) \text {, fear }(+) \text {, } \\
\text { guilt (not significant/+), animal welfare }(+)\end{array}$ \\
\hline $\begin{array}{l}\text { Wang Q., Sun J., "Consumer } \\
\text { preference and demand for organic } \\
\text { food: Evidence from a Vermont } \\
\text { survey" }\end{array}$ & 2003 & $\begin{array}{l}\text { Results suggest that there is likely a significant niche market for organic apples and } \\
\text { milk and many consumers, especially people who have purchased organic food } \\
\text { products, are willing to pay more for organic apples and milk produced locally and } \\
\text { certified by Northeast Organic Farming Association (NOFA). }\end{array}$ & $\begin{array}{l}\text { Effects on utility (choice of } \\
\text { milk/apples) } \\
\text { Organically grown (+/+) } \\
\text { Conventional (-/-) } \\
\text { NOFA certification (+/+), USDA (not } \\
\text { significant/+) } \\
\text { Not certified (-/-) }\end{array}$ & $\begin{array}{l}\text { Effects on utility (choice of milk/apples) } \\
\text { Price (-/-) }\end{array}$ \\
\hline
\end{tabular}


